https://helda.helsinki.fi

\title{
Generalized local Tb Theorems for Square Functions
}

\author{
de la Herran, Ana Grau
}

2017-01

de la Herran , A G \& Hofmann , S 2017 , ' Generalized local Tb Theorems for Square

Functions ' , Mathematika , vol. 63 , no. 1, pp. 1-28 . https://doi.org/10.1112/S0025579315000327

http://hdl.handle.net/10138/312768

https://doi.org/10.1112/S0025579315000327

acceptedVersion

Downloaded from Helda, University of Helsinki institutional repository.

This is an electronic reprint of the original article.

This reprint may differ from the original in pagination and typographic detail.

Please cite the original version. 


\title{
GENERALIZED LOCAL $T b$ THEOREMS FOR SQUARE FUNCTIONS, AND APPLICATIONS
}

\author{
ANA GRAU DE LA HERRÁN AND STEVE HOFMANN
}

\begin{abstract}
A local $T b$ theorem is an $L^{2}$ boundedness criterion by which the question of the global behavior of an operator is reduced to its local behavior, acting on a family of test functions $b_{Q}$ indexed by the dyadic cubes. We present several versions of such results, in particular, treating square function operators whose kernels do not satisfy the standard Littlewood-Paley pointwise estimates. As an application of one version of the local $T b$ theorem, we show how the solvability of the Kato problem (which was implicitly based on local $T b$ theory) may be deduced from this general criterion. We also present another version, from which we deduce boundedness of layer potentials associated to certain complex elliptic operators in divergence form.
\end{abstract}

\section{Contents}

1. Introduction, history, preliminaries

1.1. Summary of results

1.2. Acknowledgements

1.3. Notation

1.4. Some Standard Definitions

2. Local Tb Theorem for Square functions with vector-valued kernels

2.1. Step 1: Hypotheses of Theorem 2.1 imply hypotheses of Lemma $2.3 \quad 7$

2.2. Step 2: Hypotheses of Lemma2.3 imply hypotheses of Sublemma $2.4 \quad 10$

2.3. Step 3: Proof of Sublemma2.4 11

3. Local Tb Theorem without pointwise kernel bounds, version 1

3.1. Step 1: Hypotheses of Theorem 3.4 imply hypotheses of Lemma 3.8 18

3.2. Step 2: Hypotheses of Lemma 3.8 imply hypotheses of Sublemma 3.9

3.3. Step 3: Proof of Sublemma3.9 21

4. Local $\mathrm{Tb}$ Theorem without pointwise kernel bounds, version 2

5. Application to the Kato square root problem 26

6. A local $T b$ theorem with vector-valued testing functions 27

7. Application of Theorem 6.1 to the theory of layer potentials 32

8. Appendix: proof of the generalized Christ-Journé $T 1$ Theorem for square functions 


\section{INTRODUCTION, HISTORY, PRELIMINARIES}

The Tb theorem, like its predecessor, the T1 Theorem, is an $L^{2}$ boundedness criterion, originally proved by McIntosh and Meyer [ $[\mathrm{McM}]$, and by David, Journé and Semmes [DJS] in the context of singular integrals, but later extended by Semmes [Se] to the setting of "square functions". The latter arise in many applications in complex function theory and in PDE, and may be viewed as singular integrals taking values in a Hilbert space.

The essential idea of $\mathrm{Tb}$ and $\mathrm{T} 1$ type theorems, is that they reduce the question of $L^{2}$ boundedness to verifying the behavior of an operator on a single test function $\mathrm{b}$ (or even the constant function 1). The "local" versions that we have obtained are related to previous work of $\mathrm{M}$. Christ $[\mathrm{Ch}]$, who proved the first local Tb theorem in the singular integral setting. The term "local" in this context, refers to the fact that, instead of one globally defined testing function b, one is allowed to test the operator locally, say on each dyadic cube, with a local testing function that is adapted to that cube. The advantage here, in applications, is the additional flexibility that one gains: it may be easier to verify "good" behavior of the operator locally, when the testing functions are allowed to vary. The point is that sometimes particular properties of the operator may be exploited to verify the appropriate testing criterion.

Extensions of Christ's result to the non-doubling setting are due to Nazarov, Treil and Volberg [NTV] and Hytönen and Martikainen [HyM]. For doubling measures, one can also consider more general $L^{p}$ type testing conditions introduced by Auscher, Hofmann, Muscalu, Tao and Thiele [AHMTT], and further studied by Hofmann [H3], Auscher and Yan $[\mathrm{AY}]$, Auscher and Routin [AR], Hytönen and Martikainen [HyM], Hytönen and Nazarov $[\mathrm{HyN}]$, and Tan and Yan [TY].

In fact, this sort of "local Tb" criterion, in the square function setting, lies at the heart of the solution of the Kato square root problem, and was already implicit there, see [HMc], [HLMc], [AHLMcT], (and see also [AT] and [Se] for related results); the connection to local $T b$ theory is discussed in the survey article [H1]. One of the aims of the present paper is to make this connection totally explicit: that is, we prove a general version of the local $T b$ theorem for square functions from which the solution of the Kato problem follows directly. In particular, this requires that one replace pointwise size and smoothness conditions on the kernel by appropriate integral decay and orthogonality conditions.

Moreover, we further generalize the local $T b$ Theorem for square functions, to allow for relaxed size conditions on the testing functions $b_{Q}$ (i.e., scale invariant $L^{p}$ bounds, with $p>1$, rather than $p=2$ ). In particular, we extend the result of [H2] in several ways, allowing for matrix-valued $b_{Q}$ 's, and for the removal of pointwise kernel conditions. We mention that another extension (of the main result of [[H2]) of a different sort, to the setting of open sets with Ahlfors-David regular boundaries, is presented by A. Grau de la Herrán and M. Mourgoglou [GM], and has applications to problems that connect the behavior of the harmonic measure for domains with quantitative rectifiability properties of the boundary (see [HMar] and [HMarUT]).

We also present a new sort of local $T b$ theorem, one in which both the kernels of the square function, and the testing functions themselves, are vector-valued (previous results for vector-valued kernels have utilized matrix-valued testing functions.) We then apply this result to give a direct proof of a recent result of Rosen $[\mathrm{R}]$, concerning the boundedness of layer potentials associated to divergence form complex elliptic operators, in the half-space $\mathbb{R}_{+}^{n+1}$, with $t$-independent, bounded measurable coefficients. Rosen's original proof had 
relied upon functional calculus results generalizing the Kato problem (and thus also based upon local $T b$ technology), obtained in [AAMc]. Previously, such layer potential bounds had been known only for real or constant coefficients, and their perturbations [AAAHK], [HKMP.

1.1. Summary of results. We present here a brief synopsis of the results that we prove in this paper, and provide some explanatory context. The precise statements of our theorems will appear in the sequel, as noted. In these results, $\left\{\Theta_{t}\right\}$ will be a family of operators mapping $L^{2}\left(\mathbb{R}^{n}, \mathbb{C}^{m}\right), m \geq 1$, uniformly into $L^{2}\left(\mathbb{R}^{n}, \mathbb{C}\right)$, for which we seek to prove the square function bound

$$
\int_{\mathbb{R}^{n}}\left(g_{\Theta}(f)(x)\right)^{2} d x:=\iint_{\mathbb{R}_{+}^{n+1}}\left|\Theta_{t} f(x)\right|^{2} \frac{d t d x}{t} \leq C\|f\|_{L^{2}\left(\mathbb{R}^{n}\right)}^{2}
$$

Theorem 2.1. We suppose that the $\mathbb{C}^{m}$-valued kernel of $\Theta_{t}$ satisfies appropriate pointwise size and smoothness conditions, and that we are given a family $\left\{b_{Q}\right\}$ of matrix-valued testing functions, indexed by the dyadic cubes, which satisfies a scale invariant $L^{p}$ condition, for some $p>1$, and an appropriate accretivity condition. Then, given local $L^{p}$ control on $Q$, of a localized version of $g_{\Theta}\left(b_{Q}\right)$, we obtain (1.1). This theorem is stated and proved in Section 2

Theorem 3.4. We prove a version of Theorem 2.1 in which the pointwise kernel conditions are replaced by appropriate integral conditions including "off-diagonal decay" and quasi-orthogonality in $L^{2}$. In this setting, one requires local control of a conical (as opposed to vertical) square function acting on $b_{Q}$. This theorem is stated and proved in Section3

Remark. The point of Theorems 2.1 and 3.4 is that they allow for a weaker size condition ( $L^{p}$, with $p>1$ ) on the testing functions, in the vector-valued setting (i.e., with matrixvalued testing functions). Previous such results had entailed either $L^{2}$ testing conditions, or had been restricted to the scalar-valued setting; see, e.g., [H1], [H2], [H4]. In the case of Theorem 3.4, the range of $p$ allowed for the testing functions is constrained by the range of "hypercontractive" estimates enjoyed by $\Theta_{t}$.

Theorem 4.2 We prove a variant of Theorem 3.4, in which the size condition on the testing functions is strengthened to require scale invariant bounds in $L^{2}$ (and not just $L^{p}$ for some $p>1$ ), but in which quasi-othogonality is assumed to hold only on a subspace $H$ of $L^{2}\left(\mathbb{R}^{n}, \mathbb{C}^{m}\right)$. We then obtain (1.1) for $f \in H$. Here, the column vectors of the matrix valued testing functions are assumed to belong to $H$. This theorem is stated and proved in Section 4.

Theorem 5.1 We show that the solution of the Kato square root problem may be deduced as a consequence of Theorem 4.2. This theorem is stated and proved in Section 5 .

Remark. As mentioned above, local $T b$ theory was implicit in the solution of the Kato problem. Theorems 4.2 and 5.1 make this connection completely explicit.

Theorem 6.1. We prove a version of Theorem 4.2, in which the testing functions are vector-valued, rather than matrix-valued. This theorem is stated and proved in Section 6 .

Theorem 7.2 We apply Theorem 6.1 to establish $L^{2}$ bounds for layer potentials associated to a divergence form (complex) elliptic operator $L$ in $\mathbb{R}_{+}^{n+1}$, with $t$-independent coefficients, assuming that null solutions of $L$ satisfy local Hölder continuity estimates of De Giorgi/Nash type. Thus, we recover a result obtained in $[\mathbb{R}]$, but by a direct proof which 
bypasses the functional calculus formalism elaborated in [AAMc]. This theorem is stated and proved in Section 7

Remark. The novelty of Theorem 6.1 is that the testing functions are vector-valued rather than matrix-valued. This point of view turns out to be essential to our approach to the application to layer potentials. As regards Theorem 7.2 we point out that, at present, our direct treatment of layer potentials relies on estimates for the fundamental solution, proved in $[\mathrm{HK}]$ and $[\mathrm{AAAHK}]$, which assume De Giorgi/Nash type bounds. On the other hand, the results of $[\bar{R}]$ and $[\mathrm{AAMc}]$, in conjunction, show that even in the absence of De Giorgi/Nash bounds, one may define layer potentials on $L^{2}$, via the functional calculus of |AAMc]. In a forthcoming paper, we plan to extend our intrinsic development of the layer potential theory to the general case, i.e., without the De Giorgi/Nash hypothesis.

1.2. Acknowledgements. We are grateful to Tuomas Hytönen, for suggesting that we try to deduce the solution of the Kato problem directly from the general theorems obtained in the dissertation of the first named author. We also thank José María Martell, for useful discussions concerning extrapolation theory for $A_{p}$ weights, which have helped us to obtain a sharper range of exponents " $p$ " in Theorem 3.4

\subsection{Notation.}

- We shall use the letters $c, C$ to denote positive constants, not necessarily the same at each occurrence, which depend only on dimension and the constants appearing in the hypotheses of the theorems. We shall also write $A \lesssim B$ and $A \approx B$ to mean, respectively, that $A \leq C B$ and $0<c \leq A / B \leq C$, where the constants $c$ and $C$ are as above, unless explicitly noted. Moreover if we want to specify any particular dependency of the constant we will denote it by subscript or by $C(\cdot)$, e.g., $C_{n}$ or $C(n)$ is a constant that depends on dimension $n$.

- We denote points in $\mathbb{R}^{n+1}$ by $(x, t) \in \mathbb{R}^{n} \times \mathbb{R}=\mathbb{R}^{n+1}$ (we use the notational convention that $x_{n+1}=t$ ), or sometimes, for convenience, by capital letter $X$.

- We set $\mathbb{R}_{+}^{n+1}:=\mathbb{R}^{n} \times(0,+\infty)$ and $\partial \mathbb{R}_{+}^{n+1}:=\mathbb{R}^{n} \times\{0\}$.

- For a Borel set $A \subset \mathbb{R}^{n+1}$, we let $\mathbb{1}_{A}$ denote the usual indicator function of $A$, i.e. $\mathbb{1}_{A}(x)=$ 1 if $x \in A$, and $\mathbb{1}_{A}(x)=0$ if $x \notin A$.

- The letter $Q$ will be used to denote a cube in $\mathbb{R}^{n}$, and we shall write $Q_{r}$ to denote that the cube has side length $\ell(Q)=r$.

- We let $\mathbb{D}$ denote the collection of all closed dyadic cubes in $\mathbb{R}^{n}$, and let $\mathbb{D}_{k}$ denote the grid of dyadic cubes of side length $2^{-k}$.

- For a Borel set $A \subset \mathbb{R}^{n}$, a Borel measure $\mu$ defined on $\mathbb{R}^{n}$, and a Borel measurable function $f$, we set $f_{A} f d \mu=\frac{1}{\mu(A)} \int_{A} f d \mu$.

- Let $q \in[1, \infty]$, we denote by $q^{\prime} \in[1, \infty]$, the number such that we have $\frac{1}{q}+\frac{1}{q^{\prime}}=1$, where as usual we define $1 / \infty:=0$.

- For a Banach space $X$, we let $\mathcal{B}(X)$ denote the space of bounded linear operators on $X$.

\subsection{Some Standard Definitions.}

Definition 1.2. We define the Hardy Littlewood Maximal operator $\mathcal{M}$, acting on $f \in$ $L_{l o c}^{1}\left(\mathbb{R}^{n}\right)$, by

$$
\mathcal{M}(f)(x):=\sup _{r>0} \frac{1}{\left|B_{r}(x)\right|} \int_{B_{r}(x)} f(y) d y,
$$

where $B_{r}(x)$ is the ball centered at $\mathrm{x}$ and radius $\mathrm{r}$. 
Definition 1.3. We say that $P_{t}$ is a nice approximate identity, if $P_{t}$ is an operator of convolution type, with a smooth, compactly supported kernel $\Phi$. That means that for a function $f: \mathbb{R}^{n} \rightarrow \mathbb{C}$

$$
P_{t} f=\Phi_{t} * f, \text { with } \Phi_{t}=t^{-n} \Phi\left(\frac{x}{t}\right), \int \Phi(x) d x=1, \Phi_{t} \in C_{0}^{\infty}\left(\mathbb{R}^{n}\right) .
$$

In particular then, $P_{t} 1=1$, and

$$
\left(P_{t} f\right)(x) \leq C \mathcal{M} f(x)
$$

Definition 1.4. For $0<s \leq 1$, the homogeneous Sobolev space $\dot{L}_{s}^{2}$ is the completion of $C_{0}^{\infty}$ with respect to the norm $\left.\|f\|_{L_{s}^{2}}:=\|(-\Delta)^{s / 2}\right) f \|_{L^{2}}$, where $\Delta$ is the usual Laplacian.

Definition 1.5. [St2] If $0<\alpha<n$, then the Riesz potential $I_{\alpha} f$ of a locally integrable function $\mathrm{f}$ on $\mathbb{R}^{n}$ is the function defined by

$$
(-\Delta)^{-\alpha / 2} f(x)=I_{\alpha} f(x)=\frac{1}{C_{\alpha, n}} \int_{\mathbb{R}^{n}} \frac{f(y)}{|x-y|^{n-\alpha}} d y
$$

where the constant is given by $C_{\alpha, n}=\pi^{n / 2} 2^{\alpha} \frac{\Gamma(\alpha / 2)}{\Gamma((n-\alpha) / 2)}$. This fractional integral is welldefined provided $f$ decays sufficiently rapidly at infinity, specifically if $f \in L^{p}\left(\mathbb{R}^{n}\right)$ with $1 \leq p<\frac{n}{\alpha}$.

In the sequel, we shall use the following result.

Lemma 1.1. AAAHK Lemma 3.11] Suppose that $\theta_{t}$ is an operator satisfying

$$
\left\|\theta\left(f \mathbb{1}_{2^{k+1} Q \backslash 2^{k} Q}\right)\right\|_{L^{2}(Q)}^{2} \leq C 2^{-(n+2) k}\|f\|_{L^{2}\left(2^{k+1} Q \backslash 2^{k} Q\right)}
$$

whenever $t \approx \ell(Q)$, and that $\left\|\theta_{t}\right\|_{2->2} \leq C$. Let $b \in L^{\infty}\left(\mathbb{R}^{n}\right)$, and let $\mathcal{A}_{t}$ denote a self-adjoint averaging whose kernel $\varphi_{t}(x)$ satisfies $\left|\varphi_{t}(x)\right| \leq C t^{-n} \mathbb{1}_{\{|x|<C t\}}, \varphi_{t} \geq 0, \int \varphi_{t}(x) d x=1$. Then

$$
\sup _{t>0}\left\|\left(\theta_{t} b\right) \mathcal{A}_{t} f\right\|_{L^{2}\left(\mathbb{R}^{n}\right)} \leq C\|b\|_{L^{\infty}\left(\mathbb{R}^{n}\right)}\|f\|_{L^{2}\left(\mathbb{R}^{n}\right)}
$$

\section{Local Tb Theorem for SQUARe functions With Vector-VALUED Kernels}

In this section we extend the main theorem of [ [H2] to the setting where the kernels take values in $\mathbb{C}^{m}, m \geq 1$ (the case $m=1$ is the result of [[H2]). This entails that the testing functions are now matrix valued. To handle this more general situation, we follow the sectorial decomposition technique in [HLMc] and [AHLMcT], but in treating matrix valued testing functions belonging only to $L^{p}$ with $p<2$, there are certain technical difficulties which are not present either in the case $p=2$, or in the scalar case for any $p$. We let $\mathbb{M}^{m}$ denote the $m \times m$ matrices with complex entries. Here, $m$ and $n$ are not required to be equal.

Definition 2.1. Suppose that $\Psi_{t}=\left(\Psi_{t}^{1}, \ldots, \Psi_{t}^{m}\right): \mathbb{R}^{n} \times \mathbb{R}^{n} \rightarrow \mathbb{C}^{m}$ satisfies the following properties for some exponent $\alpha>0$

$$
\begin{gathered}
\left|\Psi_{t}(x, y)\right| \leq C \frac{t^{\alpha}}{(t+|x-y|)^{n+\alpha}}, \\
\left|\Psi_{t}(x, y+h)-\Psi_{t}(x, y)\right|+\left|\Psi_{t}(x+h, y)-\Psi_{t}(x, y)\right| \leq C \frac{|h|^{\alpha}}{(t+|x-y|)^{n+\alpha}},
\end{gathered}
$$


whenever $|h| \leq t / 2$. Then for vector valued $f: \mathbb{R}^{n} \rightarrow \mathbb{C}^{m}$, we define the operator

$$
\Theta_{t} f(x)=\int_{\mathbb{R}^{n}} \Psi_{t}(x, y) \cdot f(y) d y:=\sum_{j=1}^{m} \int_{\mathbb{R}^{n}} \Psi_{t}^{j}(x, y) f_{j}(y) d y .
$$

We also define $\Theta_{t}$ acting on matrix valued $b=\left(b_{i j}\right)_{1 \leq i, j \leq m}: \mathbb{R}^{n} \rightarrow \mathbb{M}^{m}$ in the obvious way, by viewing the kernel $\Psi_{t}(x, y)$ as a $1 \times m$ matrix which multiplies the $m \times m$ matrix $b$, i.e.,

$$
\Theta_{t} b(x)=\left(\sum_{i=1}^{m} \int_{\mathbb{R}^{n}} \Psi_{t}^{i}(x, y) b_{i j}(y) d y\right)_{1 \leq j \leq m} .
$$

Theorem 2.1. We define $\Theta_{t}$ as above and suppose that there exists constants $\delta>0$ and $C_{0}<\infty$, an exponent $p>1$, and a system $\left\{b_{Q}\right\} \subset L^{p}\left(\mathbb{R}^{n}, \mathbb{M}^{m}\right)$, indexed by dyadic cubes $Q \subset \mathbb{R}^{n}$, such that for each dyadic cube $Q$, we have

$$
\begin{gathered}
\int_{\mathbb{R}^{n}}\left|b_{Q}(x)\right|^{p} d x \leq C_{0}|Q| \\
\int_{Q}\left(\int_{0}^{\ell(Q)}\left|\Theta_{t} b_{Q}(x)\right|^{2} \frac{d t}{t}\right)^{\frac{p}{2}} d x \leq C_{0}|Q|, \\
\delta|\xi|^{2} \leq \operatorname{Re}\left(\xi \cdot f_{Q} b_{Q}(x) d x \bar{\xi}\right), \forall \xi \in \mathbb{C}^{m}
\end{gathered}
$$

Then

$$
\iint_{\mathbb{R}_{+}^{n+1}}\left|\Theta_{t} f(x)\right|^{2} \frac{d x d t}{t} \leq C\|f\|_{2}^{2} .
$$

The outline of the proof goes as follows. By the T1 Theorem of [CJ], Theorem 2.2, we reduce matters to showing that our operator satisfies the Carleson measure estimate 2.10 . Then the proof has three steps: 1) the conditions of Theorem 2.1 imply the conditions of Lemma 2.3, 2) the conditions of Lemma 2.3 imply the conditions of Sublemma 2.4 Finally Sublemma 2.4 establishes the Carleson measure estimate 2.10, which by the T1 theorem leads to our conclusion.

Let us first state these results, and then we will start with the proofs.

Theorem 2.2. (T1 Theorem of $[\overline{\mathrm{CJ}}])$. Let $\Theta_{t} f(x) \equiv \int_{\mathbb{R}^{n}} \Psi_{t}(x, y) \cdot f(y) d y$, where the kernel $\Psi_{t}(x, y)$ satisfies conditions (2.2) and (2.3) as above. Suppose that we have the Carleson measure estimate

$$
\sup _{Q} \frac{1}{|Q|} \int_{0}^{\ell(Q)} \int_{Q}\left|\Theta_{t} 1(x)\right|^{2} \frac{d x d t}{t} \leq C,
$$

where " 1 " in this context denotes the $m \times m$ identity matrix. Then we have the square function estimate

$$
\iint_{\mathbb{R}_{+}^{n+1}}\left|\Theta_{t} f(x)\right|^{2} \frac{d x d t}{t} \leq C\|f\|_{2}^{2}
$$

In the sequel, we shall work with cones in $\mathbb{C}^{m}$, which we identify with $\mathbb{R}^{2 m}$, having vertex at the origin. Given a unit vector $v \in \mathbb{C}^{m}$, and $\alpha>0$, we let $\Gamma^{\alpha}(v)$ denote the cone of aperture $\alpha$ and central axis $v$, i.e.,

$$
\Gamma^{\alpha}(v):=\left\{z \in \mathbb{C}^{m}:\left|\frac{z}{|z|}-v\right|<\alpha\right\}
$$


Sometimes, when working with a fixed cone, we shall simply write $\Gamma^{\alpha}$, leaving the direction vector $v$ implicit. We let $\mathbf{1}_{\Gamma^{\alpha}}$ denote the indicator function of $\Gamma^{\alpha}$, i.e., $\mathbf{1}_{\Gamma^{\alpha}}(z)=1$ if $z \in \Gamma^{\alpha}$, and $\mathbf{1}_{\Gamma^{\alpha}}(z)=0$ otherwise.

Given a small $\epsilon>0$, we cover $\mathbb{C}^{m}$ by cones of aperture $\epsilon$, enumerating these cones as $\Gamma_{1}^{\epsilon}, \ldots, \Gamma_{K}^{\epsilon}$, where $K=K(\epsilon, m)$. In the sequel, we shall also consider the "doubled" cones $\Gamma_{k}^{2 \epsilon}, 1 \leq k \leq K$, each with the same direction vector as the original one, but with the aperture $2 \epsilon$.

Lemma 2.3. Suppose that there exists $\eta \in(0,1), \epsilon>0$ small and $C_{1}<\infty$, such that for each cone $\Gamma^{\epsilon}$, and for every dyadic cube $Q \in \mathbb{R}^{n}$, there is a family $\left\{Q_{j}\right\}$ of non-overlapping dyadic sub-cubes of $Q$, satisfying

$$
\sum_{j}\left|Q_{j}\right| \leq(1-\eta)|Q|
$$

and

$$
\int_{Q}\left(\int_{\tau_{Q}(x)}^{\ell(Q)}\left|\Theta_{t} 1(x)\right|^{2} \mathbb{1}_{\Gamma^{2 \epsilon}}\left(\Theta_{t} 1(x)\right) \frac{d t}{t}\right)^{\frac{p}{2}} d x \leq C_{1}|Q|,
$$

where $\tau_{Q}(x)=\sum_{j} \ell\left(Q_{j}\right) \mathbb{1}_{Q_{j}}(x)$. Then we have the Carleson Measure estimate (2.10).

Sublemma 2.4. Suppose that $\exists N<+\infty$ and $\beta \in(0,1)$ such that for every dyadic cube $Q$, and for each cone $\Gamma^{\epsilon}$, we have

$$
\left|\left\{x \in Q: g_{Q}(x)>N\right\}\right| \leq(1-\beta)|Q|,
$$

where

$$
g_{Q}(x):=\left(\int_{0}^{\ell(Q)}\left|\Theta_{t} 1(x)\right|^{2} \mathbb{1}_{\Gamma^{2 \epsilon}}\left(\Theta_{t} 1(x)\right) \frac{d t}{t}\right)^{\frac{1}{2}} .
$$

Then we have the Carleson Measure estimate (2.10).

Remark 2.5. Every $g_{Q}$ also depends on the cone of definition but since we are choosing a generic cone we avoid complicating the notation by adding more indices.

2.1. Step 1: Hypotheses of Theorem 2.1 imply hypotheses of Lemma 2.3. We may assume without loss of generality that $1<p<2$, as the case $p>2$ may be reduced to the known case $p=2$ by Hölder's inequality. The case $p=2$ is proved in [H1].

For any cube $Q$, let

$$
R_{Q}:=Q \times(0, \ell(Q))
$$

denote the standard Carleson box above $Q$, and let $A_{t}$ denote the usual dyadic averaging operator, i.e.,

$$
A_{t} f(x):=f_{Q(x, t)} f(y) d y,
$$

where $Q(x, t)$ is the minimal dyadic cube containing $x$ with side length at least $t$.

The deep fact underlying Step 1 is the following.

Lemma 2.6. Fix a dyadic cube $Q$, a cone $\Gamma^{\epsilon}$, and its double $\Gamma^{2 \epsilon}$. Suppose that $b_{Q}$ satisfies (2.6) and (2.8). Then for $\epsilon>0$, sufficiently small, depending only on the constants $\delta$ and $C_{0}$, there is a family $\left\{Q_{j}\right\}$ of non-overlapping dyadic sub-cubes of $Q$, satisfying (2.13), such that

$$
\left|\Theta_{t} 1(x)\right|^{2} \mathbb{1}_{\Gamma^{2 \epsilon}}\left(\Theta_{t} 1(x)\right) \leq 4\left|\Theta_{t} 1(x) A_{t} b_{Q}(x)\right|^{2}, \quad \forall(x, t) \in R_{Q} \backslash\left(\bigcup_{j} R_{Q_{j}}\right) .
$$


Proof of Lemma 2.6. The proof follows that of the analogous step in the solution of the Kato square root problem (cf. [HMc], [HLMc], [AHLMcT]). We first construct the appropriate family of non-overlapping dyadic subcubes, using a stopping time argument.

Without loss of generality (by renormalizing), we may assume $\delta \equiv 1$ in (2.8) (of course, this changes $C_{0}$, depending on $\delta$ ). Fix a cube $\mathrm{Q}$, and a cone $\Gamma^{2 \epsilon}$. We subdivide $\mathrm{Q}$ dyadically and select a family $\left\{Q_{j}\right\}$ of non-overlapping dyadic subcubes of $Q$, which are maximal with respect to the property that at least one of the following conditions holds:

$$
\begin{gathered}
\frac{1}{\left|Q_{j}\right|} \int_{Q_{j}}\left|b_{Q}(x)\right| d x \geq \frac{1}{8 \epsilon} \quad \text { (type I) } \\
\operatorname{Re}\left(v \cdot f_{Q_{j}} b_{Q}(x) d x \bar{v}\right) \leq \frac{3}{4} \quad \text { (type II) }
\end{gathered}
$$

where $v$ is the unit vector in the direction of the central axis of the cone $\Gamma^{2 \epsilon}$, i.e.,

$$
\Gamma^{2 \epsilon}:=\left\{z \in \mathbb{C}^{m}:\left|\frac{z}{|z|}-v\right|<2 \epsilon\right\} .
$$

Having constructed the family, let us first verify that it satisfies the required condition (2.13). Define $E:=Q \backslash\left\{\bigcup_{j} Q_{j}\right\}$. Then from condition (2.8), since $\delta \equiv 1$, and taking $\xi=v$, we have

$$
\begin{aligned}
|Q| & \leq \operatorname{Re}\left(v \cdot \int_{Q} b_{Q}(x) d x \bar{v}\right) \\
& =\operatorname{Re}\left(v \cdot \int_{E} b_{Q}(x) d x \bar{v}\right)+\mathcal{R} e \sum_{j}\left(v \cdot \int_{Q_{j}} b_{Q}(x) d x \bar{v}\right):=I+I I .
\end{aligned}
$$

Since $v$ is a unit vector, using condition (2.6) and Hölder's inequality we get

$$
I \leq \int_{E}\left|b_{Q}(x)\right| d x \leq|E|^{\frac{1}{p^{\prime}}}\left(\int_{Q}\left|b_{Q}(x)\right|^{p} d x\right)^{\frac{1}{p}} \leq C|E|^{\frac{1}{p^{\prime}}}|Q|^{\frac{1}{p}}
$$

For the second part we separate the family of subcubes $\left\{Q_{j}\right\}$ into two cases: the ones that satisfy the type I condition and the ones that satisfy the type II condition (the same subcube can satisfy both conditions at the same time; in this case we arbitrarily assign them to be of type I). We have

$$
I I=\mathcal{R} e \sum_{j, \text { type I }}\left(v \cdot \int_{Q_{j}} b_{Q}(x) d x \bar{v}\right)+\mathcal{R} e \sum_{j, \text { type } I I}\left(v \cdot \int_{Q_{j}} b_{Q}(x) d x \bar{v}\right)=: I I_{1}+I I_{2} .
$$

Set

$$
B_{1}:=\cup_{k, \text { type I }} Q_{k}, \quad B_{2}:=\cup_{k, \text { type II }} Q_{k} .
$$

For the type I subcubes we apply Hölder's inequality, and condition (2.6), to obtain

$$
\begin{aligned}
\left|I I_{1}\right| & \leq \sum_{j, \text { type } I} \int_{Q_{j}}\left|b_{Q}(x)\right| d x=\int_{B_{1}}\left|b_{Q}(x)\right| d x \\
& \leq\left(\int_{Q}\left|b_{Q}(x)\right|^{p} d x\right)^{\frac{1}{p}}\left|B_{1}\right|^{\frac{1}{p^{\prime}}} \lesssim|Q|^{1 / p}\left|B_{1}\right|^{1 / p^{\prime}} .
\end{aligned}
$$


For the measure of $B_{1}$, by the definition of type $\mathrm{I}$, and $(2.6)$, we have that

$$
\begin{aligned}
\left|B_{1}\right|=\sum_{j, \text { type } I}\left|Q_{j}\right| & \leq \sum_{j, \text { type } I} 8 \epsilon \int_{Q_{j}}\left|b_{Q}(x)\right| d x \\
& =8 \epsilon \int_{B_{1}}\left|b_{Q}(x)\right| d x \leq 8 \epsilon\left|B_{1}\right|^{\frac{1}{p^{\prime}}}\left(\int_{Q}\left|b_{Q}(x)\right|^{p} d x\right)^{\frac{1}{p}} \\
& \lesssim 8 \epsilon\left|B_{1}\right|^{1 / p^{\prime}}|Q|^{1 / p},
\end{aligned}
$$

whence it follows that

$$
\left|B_{1}\right| \lesssim \epsilon^{p}|Q|
$$

Combining this bound with (2.20), and choosing $\epsilon$ small enough, we have

$$
\left|I I_{1}\right| \leq C \epsilon^{\frac{p}{p^{\prime}}}|Q| \leq \frac{1}{8}|Q| .
$$

By the definition of the type II cubes, we have

$$
\left|I I_{2}\right|:=\left|\mathcal{R} e \sum_{j, \text { type II }}\left(v \cdot \int_{Q_{j}} b_{Q}(x) d x \bar{v}\right)\right| \leq \frac{3}{4} \sum_{j, \text { type II }}\left|Q_{j}\right| \leq \frac{3}{4}|Q| .
$$

Combining our estimates, we obtain

$$
|Q| \leq I+I I \leq C|E|^{\frac{1}{p^{\prime}}}|Q|^{\frac{1}{p}}+\frac{1}{8}|Q|+\frac{3}{4}|Q|,
$$

whence it follows that $|Q| \leq C|E|$. We now take $0<\eta \leq \frac{1}{C}$, so that

$$
\sum_{j}\left|Q_{j}\right|=|Q \backslash E|=|Q|-|E| \leq(1-\eta)|Q|
$$

Thus, the family of cubes that we have constructed satisfies condition (2.13).

Let us now proceed to verify 2.17. We set

$$
E_{Q}^{*}:=R_{Q} \backslash\left(\bigcup_{j} R_{Q_{j}}\right)
$$

We shall prove first that

$$
\left|z \cdot A_{t} b_{Q}(x) \bar{v}\right| \geq \frac{1}{2}|z|, \quad \text { if } z \in \Gamma^{2 \epsilon} \text { and }(x, t) \in E_{Q}^{*},
$$

where we recall that $v$ is the unit direction vector for $\Gamma^{2 \epsilon}$. Indeed, if $(x, t) \in E_{Q}^{*}$, then $Q(x, t)$ is not contained in any selected cube $Q_{j}$, and therefore the dyadic average $A_{t} b_{Q}$ satisfies the opposite inequalities to those in (2.18) and (2.19). Thus, by the triangle inequality, for any unit vector $\omega \in \mathbb{C}^{m}$, we have

$$
\left|\omega \cdot A_{t} b_{Q}(x) \bar{v}\right| \geq\left|v \cdot A_{t} b_{Q}(x) \bar{v}\right|-\left|(\omega-v) A_{t} b_{Q}(x) \bar{v}\right| \geq \frac{3}{4}-|(\omega-v)| \frac{1}{8 \epsilon} .
$$

If we choose $\omega=\frac{z}{|z|}$, with $z \in \Gamma^{2 \epsilon}$, then $|\omega-v|<2 \epsilon$ (by definition of $\Gamma^{2 \epsilon}$ ), so that

$$
\left|\frac{z}{|z|} \cdot A_{t} b_{Q}(x) \bar{v}\right| \geq \frac{3}{4}-\frac{2 \epsilon}{8 \epsilon}=\frac{1}{2},
$$

which yields (2.22). We may then apply (2.22) with $z=\Theta_{t} 1(x) \in \Gamma^{2 \epsilon}$, to obtain

$$
\left|\Theta_{t} 1(x)\right|^{2} \leq 4\left|\Theta_{t} 1(x) \cdot A_{t} b_{Q}(x) \bar{v}\right|^{2} .
$$

Since $\bar{v}$ is a unit vector, we obtain (2.17), and thus also the conclusion of Lemma 2.6 
Verification of Step 1. We have already established (2.13) in Lemma 2.6. It remains to verify (2.14). To this end, we first observe that for $x \in Q$, and $\tau_{Q}(x) \leq t \leq \ell(Q)$, we have $(x, t) \in E_{Q}^{*}$. Consequently, by (2.17), we have

$$
\begin{aligned}
& \int_{Q}\left(\int_{\tau_{Q}(x)}^{\ell(Q)}\left|\Theta_{t} 1(x)\right|^{2} \mathbb{1}_{\Gamma^{2 \epsilon}}(\Theta 1(x)) \frac{d x d t}{t}\right)^{\frac{p}{2}} \\
& \leq C \int_{Q}\left(\int_{0}^{\ell(Q)}\left|\Theta_{t} 1(x) \cdot A_{t} b_{Q}(x)\right|^{2} \frac{d t}{t}\right)^{\frac{p}{2}} d x
\end{aligned}
$$

Therefore, to complete the proof of 2.14 (and thus also to complete Step 1), we are reduced to proving that

$$
\int_{Q}\left(\int_{0}^{\ell(Q)}\left|\Theta_{t} 1(x) \cdot A_{t} b_{Q}(x)\right|^{2} \frac{d t}{t}\right)^{\frac{p}{2}} d x \leq C|Q| .
$$

To this end, we use the Coifman-Meyer method and write

$$
\Theta_{t} 1 A_{t}=\left(\Theta_{t} 1\right)\left(A_{t}-P_{t}\right)+\left(\Theta_{t} 1 P_{t}-\Theta_{t}\right)+\Theta_{t}:=R_{t}^{(1)}+R_{t}^{(2)}+\Theta_{t}
$$

where $P_{t}$ is a nice approximate identity as in Definition 1.3 .

By (2.7), the contribution of $\Theta_{t} b_{Q}$ is controlled by $C|Q|$ as desired. Moreover $R_{t}^{(2)} 1=0$, and its kernel satisfies (2.2) and (2.3). Thus, by standard Littlewood-Paley/Vector-valued Calderón-Zygmund Theory, and condition (2.6), we have that

$$
\int_{Q}\left(\int_{0}^{\ell(Q)}\left|R_{t}^{(2)} b_{Q}(x)\right|^{2} \frac{d t}{t}\right)^{\frac{p}{2}} d x \leq C_{p}\left\|b_{Q}\right\|_{p}^{p} \leq C|Q|
$$

Furthermore, the same $L^{p}$ bound holds for $R_{t}^{(1)}$. Indeed, since $\theta_{t} 1$ is uniformly bounded, we may reduce matters to proving the square function bound

$$
\int_{\mathbb{R}^{n}}\left(\int_{0}^{\infty}\left|\left(A_{t}-P_{t}\right) f(x)\right|^{2} \frac{d t}{t}\right)^{\frac{p}{2}} d x \lesssim \int_{\mathbb{R}^{n}}|f(x)|^{p} d x
$$

In turn, one may establish the latter bound by following the arguments of [DRdeF]. We omit the details.

\subsection{Step 2: Hypotheses of Lemma 2.3 imply hypotheses of Sublemma 2.4.}

Proof. Fix a cone $\Gamma^{\epsilon}$, and define $g_{Q}$ as in (2.16. For a large, but fixed $\mathrm{N}$ to be chosen momentarily, let

$$
\Omega_{N}:=\left\{x \in Q: g_{Q}(x)>N\right\} .
$$


Set $E:=Q \backslash\left(\cup_{j} Q_{j}\right)$, recall that $\tau_{Q}(x):=\sum_{j} \ell\left(Q_{j}\right) \mathbb{1}_{Q_{j}}(x)$, and observe that $\tau_{Q} \equiv 0$ on $E$. If the hypotheses of Lemma 2.3 hold, then by Chebyshev's inequality we have

$$
\begin{aligned}
\left|\Omega_{N}\right| & \leq \sum_{j}\left|Q_{j}\right|+\left|\left\{x \in E: g_{Q}(x)>N\right\}\right| \\
& \leq(1-\eta)|Q|+\left|\left\{x \in E:\left(\int_{\tau_{Q}(x)}^{\ell(Q)}\left|\Theta_{t} 1(x)\right|^{2} \mathbb{1}_{\Gamma^{2 \epsilon}}\left(\Theta_{t} 1(x)\right) \mid \frac{d t}{t}\right)^{\frac{1}{2}}>N\right\}\right| \\
& \leq(1-\eta)|Q|+\frac{1}{N^{p}} \int_{Q}\left(\int_{\tau_{Q}(x)}^{\ell(Q)}\left|\Theta_{t} 1(x)\right|^{2} \mathbb{1}_{\Gamma^{2 \epsilon}}\left(\Theta_{t} 1(x)\right) \mid \frac{d t}{t}\right)^{\frac{p}{2}} d x \\
& \leq(1-\eta)|Q|+\frac{C_{1}}{N^{p}}|Q| .
\end{aligned}
$$

Choosing $\mathrm{N}$ large enough so that $\frac{C_{1}}{N^{p}} \leq \frac{\eta}{2}=: \beta$, we obtain $\left|\Omega_{N}\right| \leq(1-\beta)|Q|$.

\subsection{Step 3: Proof of Sublemma 2.4.}

Proof. Let $N, \beta$ be as in the hypotheses. Fix $\gamma \in(0,1)$, a dyadic cube Q, and a cone $\Gamma^{\epsilon}$. We first set some notation. Let

$$
\begin{aligned}
& h_{Q, \gamma}(x):=\left(\int_{\gamma}^{\min \left(\ell(Q), \frac{1}{\gamma}\right)}\left|\Theta_{t} 1(x)\right|^{2} 1_{\Gamma^{\epsilon}}\left(\Theta_{t} 1(x)\right) \frac{d t}{t}\right)^{\frac{1}{2}}, \\
& g_{Q, \gamma}(x):=\left(\int_{\gamma}^{\min \left(\ell(Q), \frac{1}{\gamma}\right)}\left|\Theta_{t} 1(x)\right|^{2} \chi_{\epsilon}\left(\Theta_{t} 1(x)\right) \frac{d t}{t}\right)^{\frac{1}{2}},
\end{aligned}
$$

where we set these terms to be 0 if $\ell(Q) \leq \gamma$, and where $\chi_{\epsilon}$ is a cut-off function adapted to the cone $\Gamma^{2 \epsilon}$, defined as follows. We let $\chi_{\epsilon}$ be homogeneous of degree zero in $\mathbb{R}^{2 m} \cong \mathbb{C}^{m}$, smooth on the sphere $\mathbb{S}^{2 m-1}$, with $0 \leq \chi_{\epsilon} \leq 1$, such that $\chi_{\epsilon}(z) \equiv 1$ on $\Gamma^{\frac{3}{2} \epsilon}$, and is supported on $\Gamma^{2 \epsilon}$. In particular,

$$
\chi_{\epsilon}\left(\Theta_{t} 1(x)\right)=\left\{\begin{array}{lll}
1 & \text { if } & \mathbb{1}_{\Gamma^{\frac{3}{2}}}\left(\Theta_{t} 1(x)\right)=1 \\
0 & \text { if } & \mathbb{1}_{\Gamma^{2 \epsilon}}\left(\Theta_{t} 1(x)\right)=0
\end{array}\right.
$$

We set

$$
k(\gamma):=\sup _{Q} \frac{1}{|Q|} \int_{Q}\left(h_{Q, \gamma}(x)\right)^{2} d x
$$

where the supremum runs over all dyadic cubes $Q$. We also define

$$
\Omega_{N, \gamma}:=\left\{x \in Q: g_{Q, \gamma}(x)>N\right\},
$$

which is an open set, by virtue of (2.3) and the fact that we have made a smooth truncation adapted to the cone $\Gamma^{\epsilon}$. Note that $k(\gamma)$ is finite for each fixed $\gamma$, and our goal is to show that $\sup _{0<\gamma<1} k(\gamma)<\infty$. We note also that $g_{Q, \gamma} \leq g_{Q}$, for every $\gamma>0$, where $g_{Q}$ is defined as in (2.16), and therefore, by (2.15),

$$
\left|\Omega_{N, \gamma}\right| \leq(1-\beta)|Q| .
$$

With this notation in place, we begin the proof. Let

$$
F_{N, \gamma}:=Q \backslash \Omega_{N, \gamma},
$$


and observe that, by (2.26), the set $F_{N, \gamma}$ is non-empty. Since $\Omega_{N, \gamma}$ is (relatively) open in $Q$, we can make a Whitney decomposition:

$$
\Omega_{N, \gamma}=\bigcup_{j} Q_{j}
$$

where the cubes $Q_{j}$ are a family of non-overlapping dyadic sub-cubes of $Q$, such that for each $Q_{j}$ in the decomposition, we have

$$
\operatorname{dist}\left(Q_{j}, F_{N, \gamma}\right) \approx \ell\left(Q_{j}\right)
$$

We warn the reader (with apologies) about a possible point of confusion: the present family $\left\{Q_{j}\right\}$ has nothing to do with the family of cubes in the statement of Lemma2.3.

We then have

$$
\begin{aligned}
& \int_{Q}\left(h_{Q, \gamma}(x)\right)^{2} d x= \int_{F_{N, \gamma}}\left(h_{Q, \gamma}(x)\right)^{2} d x+\sum_{j} \int_{Q_{j}}\left(h_{Q, \gamma}(x)\right)^{2} d x \\
& \leq \int_{F_{N, \gamma}}\left(g_{Q, \gamma}(x)\right)^{2} d x+\sum_{j} \int_{Q_{j}}\left(h_{Q_{j}, \gamma}(x)\right)^{2} d x \\
&+\sum_{j} \int_{Q_{j}} \int_{\max \left(\gamma, \ell\left(Q_{j}\right)\right)}^{\min \left(\ell(Q), \frac{1}{\gamma}\right)}\left|\Theta_{t} 1(x)\right|^{2} \mathbb{1}_{\Gamma^{\epsilon}}\left(\Theta_{t} 1(x)\right) \frac{d t d x}{t} \\
& \leq N^{2}|Q|+k(\gamma) \sum_{j}\left|Q_{j}\right|+\sum_{j} \int_{Q_{j}} \int_{\max \left(\gamma, \ell\left(Q_{j}\right)\right)}^{\min \left(\ell(Q), \frac{1}{\gamma}\right)}\left|\Theta_{t} 1(x)\right|^{2} \mathbb{1}_{\Gamma^{\epsilon}}\left(\Theta_{t} 1(x)\right) \frac{d t d x}{t} \\
& \leq N^{2}|Q|+k(\gamma)(1-\beta)|Q|+\sum_{j} \int_{Q_{j}} \int_{\max \left(\gamma, \ell\left(Q_{j}\right)\right)}^{\min \left(\ell(Q), \frac{1}{\gamma}\right)}\left|\Theta_{t} 1(x)\right|^{2} \mathbb{1}_{\Gamma^{\epsilon}}\left(\Theta_{t} 1(x)\right) \frac{d t d x}{t}
\end{aligned}
$$

We now make the following

Claim 2.7.

$$
L_{j}:=\int_{Q_{j}} \int_{\max \left(\ell\left(Q_{j}\right), \gamma\right)}^{\min \left(\ell(Q), \frac{1}{\gamma}\right)}\left|\Theta_{t} 1(x)\right|^{2} \mathbb{1}_{\Gamma^{\epsilon}}\left(\Theta_{t} 1(x)\right) \frac{d t d x}{t} \leq C\left|Q_{j}\right|
$$

Given the claim, we may divide by $|Q|$, and then take a supremum in $Q$, to obtain the uniform bound

$$
k(\gamma) \leq \frac{C_{N}}{\beta} .
$$

Therefore letting $\gamma$ approach zero we have that

$$
\int_{Q} \int_{0}^{\ell(Q)}|\Theta 1(x)|^{2} 1_{\Gamma^{\epsilon}}\left(\Theta_{t} 1(x)\right) \frac{d t d x}{t} \leq C|Q|,
$$

uniformly for all cubes $Q$ and all cones $\Gamma^{\epsilon}$. Summing over the cones $\Gamma_{k}^{\epsilon}$, we conclude that

$$
\begin{aligned}
\int_{Q} \int_{0}^{\ell(Q)}\left|\Theta_{t} 1(x)\right|^{2} \frac{d t}{t} d x & \leq \int_{Q} \int_{0}^{\ell(Q)} \sum_{k}\left|\Theta_{t} 1(x)\right|^{2} \mathbb{1}_{\Gamma_{k}^{\epsilon}}\left(\Theta_{t} 1(x)\right) \frac{d t}{t} d x \\
& \leq \sum_{k} \int_{Q} \int_{0}^{\ell(Q)}\left|\Theta_{t} 1(x)\right|^{2} \mathbb{1}_{\Gamma_{k}^{\epsilon}}\left(\Theta_{t} 1(x)\right) \frac{d t}{t} d x \\
& \leq C K(\epsilon, m)|Q|,
\end{aligned}
$$

where $K(\epsilon, m)$ is the number of cones of aperture $\epsilon$ needed to cover $\mathbb{C}^{m} \cong \mathbb{R}^{2 m}$. 
Proof of Claim 2.7 Let $0<\beta<\alpha$, where $\alpha$ is the exponent in the kernel estimates (2.2) and (2.3), and define the following sets

$$
\begin{aligned}
Q_{j}^{(1)} & :=\left\{x \in Q_{j}:\left|\Theta_{t} 1(x)\right| \leq\left(\frac{\ell\left(Q_{j}\right)}{t}\right)^{\beta} \frac{1}{\epsilon}\right\} ; \\
Q_{j}^{(2)} & :=\left\{x \in Q_{j}: \mathbb{1}_{\Gamma^{\epsilon}}\left(\Theta_{t} 1(x)\right)=0\right\} ; \\
Q_{j}^{(3)} & :=Q_{j} \backslash\left(Q_{j}^{(1)} \cup Q_{j}^{(2)}\right) .
\end{aligned}
$$

Then $L_{j} \leq L_{j}^{1}+L_{j}^{2}+L_{j}^{3}$ where

$$
L_{j}^{i}=\int_{Q_{j}^{(i)}} \int_{\max \left(\ell\left(Q_{j}\right), \gamma\right)}^{\min \left(\ell(Q), \frac{1}{\gamma}\right)}\left|\Theta_{t} 1(x)\right|^{2} \mathbb{1}_{\Gamma^{\epsilon}}\left(\Theta_{t} 1(x)\right) \frac{d t d x}{t}, \quad i=1,2,3 .
$$

Trivially, $L_{j}^{2}=0$. Moreover,

$$
L_{j}^{1} \leq \int_{Q_{j}} \int_{\ell\left(Q_{j}\right)}^{\ell(Q)}\left(\frac{\ell\left(Q_{j}\right)}{t}\right)^{\beta} \frac{1}{\epsilon} \frac{d x d t}{t} \leq C(n, \beta, \epsilon)\left|Q_{j}\right|
$$

To estimate $L_{j}^{3}$, we note that by the standard property of Whitney cubes (i.e., (2.27), to be precise), there is a point $x_{j} \in F_{N, \gamma} \operatorname{such}$ that $\operatorname{dist}\left(x_{j}, Q_{j}\right) \lesssim \ell\left(Q_{j}\right)$. We fix such an $x_{j}$, and a sufficiently large dimensional constant $C_{n}$ to be chosen, and decompose $L_{3}$ as follows:

$$
\begin{aligned}
& L_{j}^{3} \lesssim \int_{Q_{j}^{(3)}} \int_{\ell\left(Q_{j}\right)}^{C_{n} \ell\left(Q_{j}\right)}\left|\Theta_{t} 1(x)\right|^{2} \mathbb{1}_{\Gamma^{\epsilon}}\left(\Theta_{t} 1(x)\right) \frac{d t d x}{t} \\
&+\int_{Q_{j}^{(3)}} \int_{C_{n} l\left(Q_{j}\right)}^{\ell(Q)}\left|\Theta_{t} 1(x) \mathbb{1}_{\Gamma^{\epsilon}}\left(\Theta_{t} 1(x)\right)-\Theta_{t} 1\left(x_{j}\right) \chi_{\epsilon}\left(\Theta_{t} 1\left(x_{j}\right)\right)\right|^{2} \frac{d t d x}{t} \\
&+\int_{Q_{j}^{(3)}} \int_{\gamma}^{\min \left(\ell(Q), \frac{1}{\gamma}\right)}\left|\Theta_{t} 1\left(x_{j}\right)\right|^{2} \chi_{\epsilon}\left(\Theta_{t} 1\left(x_{j}\right)\right) \frac{d t d x}{t} \\
&=: I+I I+I I I .
\end{aligned}
$$

For I, we use that $\sup _{t>0}\left\|\Theta_{t} 1\right\|_{\infty}<\infty$, and for III, that $x_{j} \in F_{N, \gamma}$, so $I+I I I \leq C(n, N)\left|Q_{j}\right|$.

For II we have two cases:

Case 1: $\mathbb{1}_{\Gamma^{\epsilon}}\left(\Theta_{t} 1\left(x_{j}\right)\right)=1$. Then for $C_{n}$ large enough, by $(\underline{2.3)}$, we have

$$
\left|\Theta_{t} 1(x)-\Theta_{t} 1\left(x_{j}\right)\right| \leq C\left(\frac{\ell\left(Q_{j}\right)}{t}\right)^{\alpha}, \quad \forall x \in Q_{j} .
$$

Consequently, $I I \leq \int_{Q_{j}} \int_{C_{n} \ell\left(Q_{j}\right)}^{\infty}\left(\frac{\ell\left(Q_{j}\right)}{t}\right)^{2 \alpha} \frac{d t d x}{t} \leq C\left|Q_{j}\right|$.

Case 2: $\mathbb{1}_{\Gamma^{\epsilon}}\left(\Theta_{t} 1\left(x_{j}\right)\right)=0$. Then $\left|v-\frac{\Theta_{t} 1\left(x_{j}\right)}{\left|\Theta_{t} 1\left(x_{j}\right)\right|}\right|>\epsilon$. On the other hand, for $x \in Q_{j}^{(3)}$, we have that $\left|v-\frac{\Theta_{1} 1(x)}{\left|\Theta_{t} 1(x)\right|}\right| \leq \epsilon$, and also that $\left|\Theta_{t} 1(x)\right|>\left(\frac{\ell\left(Q_{j}\right)}{t}\right)^{\beta} \frac{1}{\epsilon}$, or equivalently, that $\frac{1}{\left|\Theta_{t} 1(x)\right|}<\left(\frac{t}{\ell\left(Q_{j}\right)}\right)^{\beta} \epsilon$. Thus, using $(2.29)$, and the elementary inequality in Remark 2.8 
below, we have for $C_{n}$ large enough, that

$$
\begin{aligned}
\left|\frac{\Theta_{t} 1(x)}{\left|\Theta_{t} 1(x)\right|}-\frac{\Theta_{t} 1\left(x_{j}\right)}{\left|\Theta_{t} 1\left(x_{j}\right)\right|}\right| \leq 2\left|\Theta_{t} 1(x)-\Theta_{t} 1\left(x_{j}\right)\right| \cdot \frac{1}{\left|\Theta_{t} 1(x)\right|} & \\
& \leq(2 C)\left(\frac{\ell\left(Q_{j}\right)}{t}\right)^{\alpha-\beta} \epsilon \leq C\left(\frac{1}{C_{n}}\right)^{\alpha-\beta} \epsilon \leq \frac{\epsilon}{2},
\end{aligned}
$$

so that

$$
\left|v-\frac{\Theta_{t} 1\left(x_{j}\right)}{\left|\Theta_{t} 1\left(x_{j}\right)\right|}\right| \leq\left|\frac{\Theta_{t} 1(x)}{\left|\Theta_{t} 1(x)\right|}-\frac{\Theta_{t} 1\left(x_{j}\right)}{\left|\Theta_{t} 1\left(x_{j}\right)\right|}\right|+\left|v-\frac{\Theta_{t} 1(x)}{\left|\Theta_{t} 1(x)\right|}\right| \leq \frac{\epsilon}{2}+\epsilon \leq \frac{3}{2} \epsilon .
$$

By the definition of the cut-off $\chi_{\epsilon}$, it then follows that

$$
I I \leq \int_{Q_{j}} \int_{C_{n} \ell\left(Q_{j}\right)}^{\ell(Q)}\left|\Theta_{t} 1(x)-\Theta_{t} 1\left(x_{j}\right)\right| \frac{d t d x}{t} \leq C\left|Q_{j}\right|,
$$

where again we have used (2.29) as in Case 1.

Remark 2.8. Observe that

$$
|(x|y|)-(y|x|)| \leq|(x|y|)-(y|y|)|+|(y|y|)-(y|x|)| \leq 2|y| \cdot|x-y|,
$$

so that

$$
\frac{|x| y|-y| x||}{|x||y|} \leq 2 \frac{|x-y|}{|x|} \Rightarrow\left|\frac{x}{|x|}-\frac{y}{|y|}\right| \leq 2 \frac{|x-y|}{|x|}
$$

3. LOCAL TB THEOREM WITHOUT POINTWISE KERNEL BOUNDS, VERSION 1

In this section, we replace the pointwise conditions on the kernel of $\Theta_{t}$, by $L^{2}$ or $L^{q}$ conditions. For testing functions in $L^{p}$, with $p<2$, this appears to require that we work with conical (local) square functions rather than vertical ones.

We begin by introducing some auxiliary operators.

Definition 3.1. We say that a family of convolution operators $\left\{Q_{s}\right\}_{s>0}$ is a CLP family ("Calderón-Littlewood-Paley" family) if for some $\sigma>0$, and some $\psi \in L^{1}\left(\mathbb{R}^{n}\right)$, with $|\psi(x)| \lesssim(1+|x|)^{-n-\sigma}$, we have

$$
\begin{aligned}
Q_{s} f=s^{-n} \psi(\cdot / s) * f, \text { and } \widehat{\psi}(\xi) & \leq C \min \left(|\xi|^{\sigma},|\xi|^{-\sigma}\right) \\
\sup _{s>0}\left(\left\|Q_{s} f\right\|_{L^{2}\left(\mathbb{R}^{n}\right)}+\left\|s \nabla Q_{s} f\right\|_{L^{2}\left(\mathbb{R}^{n}\right)}\right) & \leq C\|f\|_{L^{2}\left(\mathbb{R}^{n}\right)}, \\
\int_{\mathbb{R}^{n}} \int_{0}^{\infty}\left|Q_{s} f(x)\right|^{2} \frac{d s d x}{s} & \leq C\|f\|_{L^{2}\left(\mathbb{R}^{n}\right)}^{2}, \\
\int_{0}^{\infty} Q_{s}^{2} \frac{d s}{s} & =I,
\end{aligned}
$$

where convergence to the identity in the last formula is in the strong operator topology on $\mathcal{B}\left(L^{2}\right)$.

We also introduce some additional notation.

- Given a dyadic cube $Q$, we let $\mathbb{D}(Q)$ denote the collection of all dyadic sub-cubes of $Q$ (including, of course, $Q$ itself).

- Given a cube $Q$, we let $U_{Q}:=Q \times(\ell(Q) / 2, \ell(Q)]$ be the "Whitney box" above $Q$. 
- Given $x \in \mathbb{R}^{n}$, we define the "dyadic cone" with vertex at $x$ by

$$
\gamma^{\text {dyad }}(x):=\bigcup_{Q \in \mathbb{D}: x \in Q} U_{Q},
$$

and given a dyadic cube $Q$, for $x \in Q$, we define the "truncated dyadic cone" with vertex at $x$, of height $\ell(Q)$, by

$$
\gamma_{Q}^{\mathrm{dyad}}(x):=\bigcup_{Q^{\prime} \in \mathbb{D}(Q): x \in Q^{\prime}} U_{Q^{\prime}}
$$

Definition 3.4. We consider a family of operators $\left\{\Theta_{t}\right\}_{t>0}$, taking values in $\mathbb{C}^{m}, m \geq 1$, so that $\Theta_{t}:=\left(\Theta_{t}^{1}, \Theta_{t}^{2}, \ldots, \Theta_{t}^{m}\right)$, and for $f=\left(f^{1}, f^{2}, \ldots, f^{m}\right) \in L^{2}\left(\mathbb{R}^{n}, \mathbb{C}^{m}\right)$, we set

$$
\Theta_{t} f:=\sum_{j=1}^{m} \Theta_{t}^{j} f^{j}
$$

We also define the action of $\Theta_{t}$ on an $m \times m$ matrix valued function $b=\left(b_{j, k}\right)$, in the obvious way, i.e., $\Theta_{t} b=\left(\left(\Theta_{t} b\right)_{1},\left(\Theta_{t} b\right)_{2}, \ldots,\left(\Theta_{t} b\right)_{m}\right)$ is a $\mathbb{C}^{m}$ valued function, with

$$
\left(\Theta_{t} b\right)_{k}:=\sum_{j=1}^{m} \Theta_{t}^{j} b_{j, k} .
$$

We suppose that $\Theta_{t}$ satisfies the following properties:

(a) (Uniform $L^{2}$ bounds and off-diagonal decay in $L^{2}$ ).

$$
\begin{gathered}
\sup _{t>0}\left\|\Theta_{t} f\right\|_{L^{2}\left(\mathbb{R}^{n}\right)} \leq C\|f\|_{L^{2}\left(\mathbb{R}^{n}\right)}, \\
\left\|\Theta_{t} f_{j}\right\|_{L^{2}(Q)} \leq C 2^{-j(n+2+\beta) / 2}\left\|f_{j}\right\|_{L^{2}\left(2^{j+1} Q \backslash 2^{j} Q\right)}, \quad \ell(Q) \leq t \leq 2 \ell(Q),
\end{gathered}
$$

for some $\beta>0$, where $f_{j}:=f \mathbb{1}_{2^{j+1}} Q \backslash 2^{j} Q$.

(b) (Quasi-orthogonality in $L^{2}$ ). For some (hence every) CLP family $\left\{Q_{s}\right\}$, there is a $\beta>0$ for which we have

$$
\left\|\Theta_{t} Q_{s} h\right\|_{L^{2}\left(\mathbb{R}^{n}\right)} \leq C\left(\frac{s}{t}\right)^{\beta}\|h\|_{L^{2}\left(\mathbb{R}^{n}\right)}, \quad \forall s \leq t .
$$

(c) ("Hypercontractive" off-diagonal decay). There is some $1<r<2$, and some $\mu>\frac{n}{r}$ $\left(\mu=\frac{n}{r}+\varepsilon\right.$, for some $\left.\varepsilon>0\right)$, such that

$$
\begin{aligned}
&\left(\int_{Q^{*}}\left|\Theta_{t}\left(f \mathbb{1}_{S_{j}}(Q)\right)(y)\right|^{2} d y\right)^{\frac{1}{2}} \leq C 2^{-j \mu} t^{-n\left(\frac{1}{r}-\frac{1}{2}\right)}\left(\int_{S_{j}(Q)}|f(y)|^{r} d y\right)^{\frac{1}{r}}, \\
& \forall j \geq 0, \ell(Q)<t \leq 2 \ell(Q),
\end{aligned}
$$

where $S_{0}(Q)=16 Q$ and $S_{j}(Q)=2^{j+4} Q \backslash 2^{j+3} Q, j \geq 1 . Q^{*} \equiv 8 Q$.

(d) (Improved integrability). There is an exponent $q>2$ such that

$$
\sup _{t>0}\left\|\Theta_{t} f\right\|_{L^{q}\left(\mathbb{R}^{n}\right)} \leq C\|f\|_{L^{q}\left(\mathbb{R}^{n}\right)} .
$$

Remark 3.1. If (3.6) holds for all $t>0$ (not just for $t \approx \ell(Q)$ ), as is often the case in applications, then hypothesis (d) is redundant, and in fact (3.9) holds for all $q>2$; indeed, one has the pointwise bound

$$
\mathcal{M}\left(\Theta_{t} f\right) \lesssim\left(\mathcal{M}\left(|f|^{2}\right)\right)^{1 / 2} .
$$


We leave the details to the interested reader.

Remark 3.2. We observe that, for example, (b), (c), and (d) hold, with $\theta_{t}=t \partial_{t} P_{t}$, where $P_{t}=e^{-t \sqrt{-\Delta}}$ is the usual Poisson semigroup, and that (a) holds with $\beta=0$, for the same operator. We may obtain a positive value of $\beta$ in (a), by considering higher order derivatives of $P_{t}$. As a practical matter, when considering square functions arising in PDE applications, it is often a fairly routine matter to pass to higher order derivatives. The advantage of the present formulation of our conditions, is that these conditions may continue to hold in the absence of pointwise kernel bounds. In PDE applications, (d) is typically obtained as a consequence of higher integrability estimates of "N. Meyers" type (cf. [Me2]).

We shall need to work with some "dyadic conical" square functions, and their local analogues.

Definition 3.10. Given $F$ with domain $\mathbb{R}_{+}^{n+1}$, we define the "dyadic conical square function" of $F$ by

$$
\mathcal{A}^{\text {dyad }}(F)(x):=\left(\iint_{\gamma^{\text {dyad }}(x)}|F(y, t)|^{2} \frac{d y d t}{t^{n+1}}\right)^{1 / 2} .
$$

Similarly, we define the "truncated dyadic conical square function" relative to a dyadic cube $Q$ by

$$
\mathcal{A}_{Q}^{\text {dyad }}(F)(x):=\left(\iint_{\gamma_{Q}^{\text {dyad }}(x)}|F(y, t)|^{2} \frac{d y d t}{t^{n+1}}\right)^{1 / 2} .
$$

Remark 3.3. We observe that $\mathcal{A}_{Q}^{\text {dyad }}(F)$ is dominated by the standard local square function

$$
\mathcal{A}_{Q}^{\alpha}(F)(x):=\left(\int_{0}^{\ell(Q)} \int_{|x-y|<\alpha t}|F(y, t)|^{2} \frac{d y d t}{t^{n+1}}\right)^{1 / 2},
$$

assuming that the aperture $\alpha$ is chosen large enough, as may be seen from the definition of the dyadic cones, and a trivial geometric argument.

As before, for $\epsilon$ small but fixed, we cover $\mathbb{C}^{m}$ by cones $\Gamma_{k}^{\epsilon}$, of aperture $\epsilon$, with vertex at the origin. The constants in our estimates are then allowed to depend on $K=K(\epsilon, m)$, the number of cones in the covering. Given any such cone $\Gamma^{\epsilon}$, we shall also need to consider dyadic conical square functions "restricted" to the doubled cone $\Gamma^{2 \epsilon}$ :

$$
\mathcal{A}_{Q, \Gamma^{\epsilon}}^{\text {dyad }}(F)(x):=\left(\iint_{\gamma_{Q}^{\text {dyad }}(x)}|F(y, t)|^{2} \mathbb{1}_{\Gamma^{\epsilon}}(F(y, t)) \frac{d y d t}{t^{n+1}}\right)^{1 / 2} .
$$

Our main result in this section is the following:

Theorem 3.4. Let $\left\{\Theta_{t}\right\}_{t>0}$, be as in Definition 3.4 above, and suppose that there exist positive constants $C_{0}<\infty$, and $\delta>0$, an exponent $p>r$, and a system $\left\{b_{Q}\right\}$ of complex $m \times m$ matrix-valued functions indexed by dyadic cubes $Q \subset \mathbb{R}^{n}$, such that for each dyadic cube $Q$ :

$$
\begin{gathered}
\int_{\mathbb{R}^{n}}\left|b_{Q}(x)\right|^{p} d x \leq C_{0}|Q|, \\
\int_{Q}\left(\mathcal{A}_{Q}^{\mathrm{dyad}}\left(\Theta_{t} b_{Q}\right)(x)\right)^{p} d x \leq C_{0}|Q|,
\end{gathered}
$$




$$
\delta|\xi|^{2} \leq \operatorname{Re}\left(\xi \cdot f_{Q} b_{Q}(x) d x \bar{\xi}\right), \quad \forall \xi \in \mathbb{C}^{m}
$$

Then

$$
\iint_{\mathbb{R}_{+}^{n+1}}\left|\Theta_{t} f(x)\right|^{2} \frac{d x d t}{t} \leq C\|f\|_{L^{2}\left(\mathbb{R}^{n}\right)}^{2},
$$

Remark 3.5. We observe that by Remark 3.3 , we could replace $\mathcal{A}_{Q}^{\text {dyad }}$ by $\mathcal{A}_{Q}^{\alpha}$, with $\alpha$ sufficiently large, in hypothesis (3.13).

We begin with a generalization of the Christ-Journé $T 1$ theorem for square functions.

Theorem 3.6. (T1 Theorem) Suppose that $\Theta_{t} f(x)$ satisfies conditions (3.5), (3.6) and (3.7) as above, and also the Carleson measure estimate

$$
\sup _{Q} \frac{1}{|Q|} \int_{Q} \int_{0}^{\ell(Q)}\left|\Theta_{t} 1(x)\right|^{2} \frac{d x d t}{t} \leq C .
$$

Then we have the square function estimate

$$
\iint_{\mathbb{R}_{+}^{n+1}}\left|\Theta_{t} f(x)\right|^{2} \frac{d x d t}{t} \leq C\|f\|_{L^{2}\left(\mathbb{R}^{n}\right)}^{2}
$$

Remark 3.7. Here, the constant function 1 should be interpreted in the matrix-valued sense, i.e., as the $m \times m$ identity matrix.

We defer the proof of Theorem 3.6 to an appendix (Section 8).

Lemma 3.8. Suppose that there exists $\eta \in(0,1), \epsilon>0$ small and $C<\infty$ such that for every dyadic cube $Q \in \mathbb{R}^{n}$, and for each cone $\Gamma^{\epsilon}$ of aperture $\epsilon$, there is a family $\left\{Q_{j}\right\}_{j}$ of non-overlapping dyadic sub-cubes of $Q$, with

$$
\sum_{j}\left|Q_{j}\right| \leq(1-\eta)|Q|
$$

and

where $E:=Q \backslash\left\{\cup_{j} Q_{j}\right\}$. Then

$$
\int_{E}\left(\mathcal{A}_{Q, \Gamma^{\epsilon}}^{\mathrm{dyad}}\left(\Theta_{t} 1\right)(x)\right)^{p} d x \leq C|Q|
$$

$$
\sup _{Q} \frac{1}{|Q|} \int_{0}^{\ell(Q)} \int_{Q}\left|\Theta_{t} 1(x)\right|^{2} \frac{d x d t}{t} \leq C
$$

Sublemma 3.9. Suppose that there exist $N<+\infty$, and $\beta \in(0,1)$, such that for every cube $Q$ and for each cone $\Gamma^{\epsilon}$

$$
\left|\left\{x \in Q: G_{Q}(x)>N\right\}\right| \leq(1-\beta)|Q|
$$

where

Then

$$
G_{Q}:=\mathcal{A}_{Q, \Gamma^{\epsilon}}^{\mathrm{dyad}}\left(\Theta_{t} 1\right)
$$

$$
\sup _{Q} \frac{1}{|Q|} \int_{0}^{\ell(Q)} \int_{Q}\left|\Theta_{t} 1(x)\right|^{2} \frac{d x d t}{t} \leq C
$$

The scheme of our proof now follows the three-step argument of Section 2 . 


\subsection{Step 1: Hypotheses of Theorem 3.4 imply hypotheses of Lemma 3.8.}

Proof. We fix a dyadic cube $Q$, and a cone

$$
\Gamma^{\epsilon}=\left\{z \in \mathbb{C}^{m}:\left|\frac{z}{|z|}-v\right|<\epsilon\right\},
$$

with aperture $\epsilon$, and unit direction vector $v \in \mathbb{C}^{m}$. As above, we define the usual dyadic averaging operator, by setting $A_{t} f(x):=|Q(x, t)|^{-1} \int_{Q(x, t)} f(y) d y$, where $Q(x, t)$ is the minimal dyadic sub-cube of $Q$, containing $\mathrm{x}$, with side length at least $\mathrm{t}$.

Assuming the hypotheses of Theorem 3.4 we then obtain by Lemma 2.6 above, that for $\epsilon$ small enough, we can construct the required family $\left\{Q_{j}\right\}$ of dyadic sub-cubes of $Q$, satisfying (3.18), as well as

$$
\left|\Theta_{t} 1(x)\right|^{2} \mathbb{1}_{\Gamma^{\epsilon}}\left(\Theta_{t} 1(x)\right) \leq 4\left|\Theta_{t} 1(x) A_{t} b_{Q}(x)\right|^{2}, \quad \forall(x, t) \in E_{Q}^{*}:=R_{Q} \backslash\left(\bigcup_{j} R_{Q_{j}}\right) .
$$

With (3.23) in hand, we may now verify the second condition of the lemma, (3.19). We first note that for $x \in E=Q \backslash\left(\cup_{j} Q_{j}\right)$, if $x \in Q^{\prime} \in \mathbb{D}(Q)$, then $Q^{\prime}$ is not contained in any $Q_{j}$. Thus, for $x \in E$, by definition of the dyadic cones, we have $\gamma_{Q}^{\text {dyad }}(x) \subset E_{Q}^{*}$. Consequently, by (3.23),

$$
\int_{E}\left(\mathcal{A}_{Q, \Gamma^{\epsilon}}^{\mathrm{dyad}}\left(\Theta_{t} 1\right)\right)^{p} d x \lesssim \int_{Q}\left(\iint_{\gamma_{Q}^{\text {dyad }}(x)}\left|\Theta_{t} 1(y) A_{t} b_{Q}(y)\right|^{2} \frac{d y d t}{t^{n+1}}\right)^{p / 2} d x .
$$

We now use the Coifman-Meyer trick to write

$$
\begin{aligned}
\Theta_{t} 1 A_{t} b_{Q}=\left(\left(\Theta_{t} 1\right) A_{t}-\left(\Theta_{t} 1\right) A_{t} P_{t}\right) b_{Q}+\left(\left(\Theta_{t} 1\right) A_{t} P_{t}-\Theta_{t}\right) b_{Q}+\Theta_{t} b_{Q} \\
=: R_{t}^{(1)} b_{Q}+R_{t}^{(2)} b_{Q}+\Theta_{t} b_{Q},
\end{aligned}
$$

where as usual $P_{t}$ denotes a nice approximate identity operator (cf. Definition 1.3 above). By (3.13), the contribution of the term $\Theta_{t} b_{Q}$ gives the desired bound.

To treat the two "remainder terms" $R_{t}^{(1)} b_{Q}$ and $R_{t}^{(2)} b_{Q}$, we first recall the following.

Proposition 3.10. [C-UMP Let $T$ be a sublinear operator satisfying

$$
T: L^{2}\left(\mathbb{R}^{n}, v\right) \rightarrow L^{2}\left(\mathbb{R}^{n}, v\right), \quad \forall v \in A_{2 / r} .
$$

Then $T: L^{p}\left(\mathbb{R}^{n}\right) \rightarrow L^{p}\left(\mathbb{R}^{n}\right)$, for $p>r$.

By the Proposition, and (3.12), we are left to prove, for $i=1,2$, that

$$
\int_{\mathbb{R}^{n}}\left|\mathcal{A}^{\mathrm{dyad}}\left(R_{t}^{(i)} f\right)(x)\right|^{2} v(x) d x \leq C \int_{\mathbb{R}^{n}}|f(x)|^{2} v(x) d x, \quad v \in A_{2 / r} .
$$

By definition of the dyadic cones used to construct $\mathcal{A}^{\text {dyad }}$, the left side of (3.25) equals

$$
\int_{\mathbb{R}^{n}} \sum_{Q \in \mathbb{D}} 1_{Q}(x) \iint_{U_{Q}}\left|R_{t}^{(i)} f(y)\right|^{2} \frac{d y d t}{t^{n+1}} v(x) d x \leq \int_{0}^{\infty} \int_{\mathbb{R}^{n}} t^{-n} \int_{|x-y|<C t}\left|R_{t}^{(i)} f(y)\right|^{2} d y v(x) d x \frac{d t}{t}
$$

Thus, by a standard orthogonality argument, it suffices to prove that for some $\beta_{1}>0$, we have

$$
\int_{\mathbb{R}^{n}} t^{-n} \int_{|x-y|<C t}\left|R_{t}^{(i)} Q_{s} h(y)\right|^{2} d y v(x) d x \leq C \min \left(\frac{s}{t}, \frac{t}{s}\right)^{\beta_{1}} \int_{\mathbb{R}^{n}}|h(x)|^{2} v(x) d x,
$$


where $\left\{Q_{s}\right\}_{s>0}$ is a CLP family as in Definition 3.1, which in addition satisfies the weighted $L^{2}$ estimate

$$
\int_{0}^{\infty} \int_{\mathbb{R}^{n}}\left|Q_{s} f(x)\right|^{2} v(x) \frac{d x d t}{t} \leq C \int_{\mathbb{R}^{n}}|f(x)|^{2} v(x) d x, \quad v \in A_{2 / r} .
$$

To this end, we begin by showing that (3.26) holds in the unweighted case $v \equiv 1$, i.e., that for some $\beta_{0}>0$, we have

$$
\int_{\mathbb{R}^{n}} t^{-n} \int_{|x-y|<C t}\left|R_{t}^{(i)} Q_{s} h(y)\right|^{2} d y d x \approx \int_{\mathbb{R}^{n}}\left|R_{t}^{(i)} Q_{s} h(y)\right|^{2} d y \lesssim \min \left(\frac{s}{t}, \frac{t}{s}\right)^{\beta_{0}} \int_{\mathbb{R}^{n}}|h(x)|^{2} d x .
$$

In fact, more generally, we shall prove the following

Lemma 3.11. Let $H$ be a subspace of $L^{2}\left(\mathbb{R}^{n}, \mathbb{C}^{m}\right), m \geq 1$, and suppose that $\Theta_{t}$ satisfies (3.5), (3.6), and, for every $h \in H$, (3.7). Let $R_{t}^{(i)}, i=1,2$, be defined as in 3.24). Then there is some $\beta_{0}>0$, such that the bound (3.27) holds for all $h \in H$, and for $i=1,2$.

We remark that for our purposes at present, we simply take $H$ to be all of $L^{2}\left(\mathbb{R}^{n}, \mathbb{C}^{m}\right)$, but we record the more general version stated above, as well as the following Corollary, for future reference.

Corollary 3.12. Under the hypotheses of Lemma 3.11 for $i=1,2$, we have the square function bound

$$
\iint_{\mathbb{R}_{+}^{n+1}}\left|R_{t}^{(i)} h(x)\right|^{2} \frac{d x d t}{t} \leq C\|h\|_{2}^{2}, \quad \forall h \in H .
$$

The Corollary follows from the Lemma by a standard orthogonality argument of "Schur's lemma" type. We omit the routine details.

Proof of Lemma 3.11 We note that by (3.5) and (3.6), we may invoke AAAHK, Lemma 3.11] (cf. Lemma 1.1 above), to deduce that $\Theta_{t} 1$ is well defined, and satisfies

$$
\sup _{t>0}\left\|\left(\Theta_{t} 1\right) A_{t}\right\|_{L^{2}\left(\mathbb{R}^{n}\right) \rightarrow L^{2}\left(\mathbb{R}^{n}\right)} \leq C,
$$

and therefore also that each $R_{t}^{(i)}$ satisfies (3.5) and (3.6). Thus, since in addition, we have that $R_{t}^{(i)} 1=0$ for $i=1,2$, we may invoke [AAAHK, Lemma 3.5], to obtain 1 that

$$
\left\|R_{t}^{(i)} Q_{s} h\right\|_{2} \lesssim t\left\|\nabla Q_{s} h\right\|_{2}, \quad \forall h \in L^{2},
$$

which yields (3.27), in the case $t \leq s$, with $\beta_{0}=2$, by properties of $Q_{s}$ (cf. Definition 3.1).

Consider now the case $s \leq t$ of 3.27). Since $A_{t}$ is a projection operator, we have

$$
R_{t}^{(1)}=\left(\Theta_{t} 1\right) A_{t}\left(A_{t}-P_{t}\right),
$$

so that the desired bound for this term, for all $h \in L^{2}$, follows from (3.28), and the well known fact that there is some $\mu>0$ such that

$$
\left\|A_{t} Q_{s}\right\|_{L^{2} \rightarrow L^{2}}+\left\|P_{t} Q_{s}\right\|_{L^{2} \rightarrow L^{2}} \lesssim\left(\frac{s}{t}\right)^{\mu}, \quad s \leq t .
$$

Similarly, the case $s \leq t$ of 3.27 follows for $R_{t}^{(2)}$ from the latter fact, and the fact that (3.7) holds for all $h \in H$.

\footnotetext{
${ }^{1}$ AAAHK Lemma 3.11 and Lemma 3.5] are stated for operators satisfying our off-diagonal decay estimate 3.6 for all $t \lesssim \ell(Q)$, but the proof in [AAAHK actually requires only that off-diagonal decay bounds hold with $t \approx \ell(Q)$. Also [AAAHK Lemma 3.5] is stated under the hypothesis that [3.6 holds with $\beta \geq 2$, but the proof given there actually requires only that $\beta>0$, as we assume here.
} 
With 3.27) in hand, we return to the proof of (3.26), and we make the following claim:

\section{Claim 3.13.}

$$
\int_{\mathbb{R}^{n}} t^{-n} \int_{|x-y|<C t}\left|R_{t}^{(i)} Q_{s} h(y)\right|^{2} d y \tilde{v}(x) d x \leq C \int_{\mathbb{R}^{n}}|h(x)|^{2} \tilde{v}(x) d x, \quad \forall \tilde{v} \in A_{2 / r}
$$

with $i=1,2$.

Interpolating with change of measure ([SW]) between (3.27) and (3.29), we get 3.26). Indeed, for each $v \in A_{\frac{2}{r}}$, there exist $\tau>0$ such that $v^{1+\tau} \in A_{\frac{2}{r}}$, so we choose $\tilde{v}(x)=v^{1+\tau}(x)$. To our knowledge, the idea of using interpolation with change of measure in this way first appeared in the paper [DRdeF]. Modulo the proof of the claim, Step 1 is now complete.

Proof of Claim 3.13. Define $\tilde{h}(x)=Q_{s} h(x)$. By properties of $Q_{s}$ (it is controlled by the maximal operator), and since $A_{2 / r} \subset A_{2}$, we have $\|\tilde{h}\|_{L_{\tilde{r}}^{2}\left(\mathbb{R}^{n}\right)} \leq C\|h\|_{L_{\tilde{v}}^{2}\left(\mathbb{R}^{n}\right)}$. Thus, it is enough to prove

$$
\int_{\mathbb{R}^{n}} t^{-n} \int_{|x-y|<C t}\left|R_{t} \tilde{h}(y)\right|^{2} d y \tilde{v}(x) d x \leq C \int_{\mathbb{R}^{n}} \tilde{h}(x)^{2} \tilde{v}(x) d x
$$

For $t>0$, let $\mathbb{D}(t)$ denote the grid of dyadic cubes $P \subset \mathbb{R}^{n}$ with length $\ell(P) \in(t / 2, t]$, and let $P^{*}$ denote the concentric dilate $P^{*}=\kappa P$ for some large $\kappa$. Recall that $S_{j}(P):=$ $2^{j+4} P \backslash 2{ }^{j+3} P$. By property (c) of Definition 3.4 , and since $\tilde{v} \in A_{2 / r}$ (which gives us an $L_{\tilde{v}}^{2 / r}$ bound for the maximal function), we have

$$
\begin{gathered}
\left(\int_{\mathbb{R}^{n}} t^{-n} \int_{|x-y|<C t}\left|R_{t}^{(i)} \tilde{h}(y)\right|^{2} d y \tilde{v}(x) d x\right)^{1 / 2}=\left(\sum_{P \in \mathbb{D}(t)} \int_{P} t^{-n} \int_{|x-y|<C t}\left|R_{t}^{(i)} \tilde{h}(y)\right|^{2} d y \tilde{v}(x) d x\right)^{1 / 2} \\
\leq C\left(\sum_{P \in \mathbb{D}(t)} \int_{P} \frac{1}{\left|P^{*}\right|} \int_{P^{*}}\left|R_{t}^{(i)} \tilde{h}(y)\right|^{2} d y \tilde{v}(x) d x\right)^{1 / 2} \\
\leq C \sum_{j=0}^{\infty}\left(\sum_{P \in \mathbb{D}(t)} \int_{P} \frac{1}{\left|P^{*}\right|} \int_{P^{*}}\left|R_{t}^{(i)}\left(\tilde{h} \mathbb{1}_{S_{j}(P)}\right)(y)\right|^{2} d y \tilde{v}(x) d x\right)^{1 / 2} \\
\leq C \sum_{j=0}^{\infty}\left(\sum_{P \in \mathbb{D}(t)} \int_{P} \frac{1}{\left|P^{*}\right|} 2^{-2 j \mu} t^{-n\left(\frac{2}{r}-1\right)}\left(\int_{S_{j}(P)}|\tilde{h}(y)|^{r} d y\right)^{2 / r} \tilde{v}(x) d x\right)^{1 / 2}
\end{gathered}
$$

where in the last step, we have used that by (3.28), we may apply (3.8) to $R_{t}^{(i)}$, since $A_{t}$ is a projection, and since $A_{t}$ and $P_{t}$ have compactly supported kernels. In turn, since $\left|P^{*}\right| \approx t^{n}$, 
and since $\mu=n / r+\varepsilon$, the last expression in 3.30) is comparable to

$$
\begin{gathered}
\sum_{j=0}^{\infty}\left(\sum_{P \in \mathbb{D}(t)} \int_{P} 2^{-2 j \epsilon} 2^{-2 j n / r} t^{-2 n / r} \tilde{v}(x)\left(\int_{S_{j}(P)}|\tilde{h}(y)|^{r} d y\right)^{2 / r} d x\right)^{1 / 2} \\
\approx \sum_{j=0}^{\infty}\left(\sum_{P \in \mathbb{D}(t)} \int_{P} 2^{-2 j \epsilon} \tilde{v}(x)\left(f_{S_{j}(P)}|\tilde{h}(y)|^{r} d y\right)^{2 / r} d x\right)^{1 / 2} \\
\lesssim \sum_{j=0}^{\infty} 2^{-j \epsilon}\left(\sum_{P \in \mathbb{D}(t)} \int_{P}\left(\mathcal{M}\left(|\tilde{h}(x)|^{r}\right)\right)^{\frac{2}{r}} \tilde{v}(x) d x\right)^{1 / 2} \\
\lesssim \sum_{j=0}^{\infty} 2^{-j \epsilon}\left(\int_{\mathbb{R}^{n}}|\tilde{h}(x)|^{2} \tilde{v}(x) d x\right)^{1 / 2} \approx\left(\int_{\mathbb{R}^{n}}|\tilde{h}(x)|^{2} \tilde{v}(x) d x\right)^{1 / 2},
\end{gathered}
$$

where in the next-to-last step, we have used that the maximal operator $\mathcal{M}$ is bounded on $L_{v}^{2 / r}$, since $v \in A_{2 / r}$. The proof of Claim 3.13 is now complete.

\subsection{Step 2: Hypotheses of Lemma 3.8 imply hypotheses of Sublemma 3.9.}

Proof. Recall that

$$
G_{Q}:=\mathcal{A}_{Q, \Gamma^{\epsilon}}^{\text {dyad }}\left(\Theta_{t} 1\right)
$$

For a large, but fixed $N$ (to be chosen momentarily) let $\Omega_{N}:=\left\{x \in Q: G_{Q}(x)>N\right\}$. If the hypotheses of the lemma hold with $E=Q \backslash \bigcup_{j} Q_{j}$, we then have

$$
\begin{aligned}
\left|\Omega_{N}\right| & \leq \sum_{j}\left|Q_{j}\right|+\left|\left\{x \in E: G_{Q}(x)>N\right\}\right| \\
& \leq(1-\eta)|Q|+\frac{1}{N^{p}} \int_{E}\left(\mathcal{A}_{Q, \Gamma^{\epsilon}}^{\mathrm{dyad}}\left(\Theta_{t} 1\right)(x)\right)^{p} d x \\
& \leq\left[(1-\eta)+\frac{C}{N^{p}}\right]|Q| \leq(1-\beta)|Q|,
\end{aligned}
$$

for some $\beta>0$, where we obtain the last estimate by choosing $N$ large enough, depending on $\eta$.

\subsection{Step 3: Proof of Sublemma 3.9.}

Proof. Fix (momentarily) a large $M$, so that $2^{-M} \in(0,1)$, and given a dyadic cube $Q$, set

$$
\mathbb{D}_{M}(Q):=\left\{Q^{\prime} \in \mathbb{D}(Q): 2^{-M} \leq \ell\left(Q^{\prime}\right) \leq 2^{M}\right\}
$$

(of course, the upper bound on $\ell\left(Q^{\prime}\right)$ is relevant only if $\ell(Q)>2^{M}$ ). Define a truncated dyadic cone

$$
\gamma_{Q, M}^{\mathrm{dyad}}(x):=\bigcup_{Q^{\prime} \in \mathbb{D}_{M}(Q): x \in Q^{\prime}} U_{Q^{\prime}}
$$


For each dyadic cube $Q$, set

$$
G_{Q, M}(x):=\left(\iint_{\gamma_{Q, M}^{\mathrm{dyad}}(x)}\left|\Theta_{t} 1(y)\right|^{2} \mathbb{1}_{\Gamma^{\epsilon}}\left(\Theta_{t} 1(y)\right) \frac{d y d t}{t^{n+1}}\right)^{1 / 2} .
$$

Thus, by definition, $G_{Q, M} \leq G_{Q}:=\mathcal{A}_{Q, \Gamma^{\epsilon}}^{\text {dyad }}\left(\Theta_{t} 1\right)$, and we note that $G_{Q, M} \rightarrow G_{Q}$, as $M \rightarrow \infty$, by monotone convergence. Now let $N, \beta$ be as in the hypotheses. Setting

$$
\Omega_{N, M}=\Omega_{N, M}(Q):=\left\{x \in Q: G_{Q, M}>N\right\},
$$

we have that

$$
\left|\Omega_{N, M}\right| \leq\left|\left\{x \in Q: G_{Q}>N\right\}\right| \leq(1-\beta)|Q|
$$

by hypothesis. We set

$$
K(M):=\sup _{Q} \frac{1}{|Q|} \int_{Q}\left(G_{Q, M}(x)\right)^{2} d x .
$$

We observe that, for each fixed $M, K(M)$ is finite, by the truncation of the cones defining $G_{Q, M}$. Our goal is to show that

$$
\sup _{M<\infty} K(M):=K_{0}<\infty .
$$

Indeed, in that case, letting $M \rightarrow \infty$, we then obtain by monotone convergence that, for every cube $Q$,

$$
\begin{gathered}
K_{0}|Q| \geq \int_{Q}\left(G_{Q^{\prime}}(x)\right)^{2} d x=\int_{Q} \iint_{\gamma_{Q}^{\mathrm{dyad}}(x)}\left|\Theta_{t} 1(y)\right|^{2} \mathbb{1}_{\Gamma^{\epsilon}}\left(\Theta_{t} 1(y)\right) \frac{d y d t}{t^{n+1}} d x \\
=\int_{Q} \sum_{Q^{\prime} \in \mathbb{D}(Q)} 1_{Q^{\prime}}(x) \iint_{U_{Q^{\prime}}}\left|\Theta_{t} 1(y)\right|^{2} \mathbb{1}_{\Gamma^{\epsilon}}\left(\Theta_{t} 1(y)\right) \frac{d y d t}{t^{n+1}} d x \\
=\sum_{Q^{\prime} \in \mathbb{D}(Q)} \int_{\ell\left(Q^{\prime}\right) / 2}^{\ell\left(Q^{\prime}\right)} \int_{Q^{\prime}}\left|\Theta_{t} 1(y)\right|^{2} \mathbb{1}_{\Gamma^{\epsilon}}\left(\Theta_{t} 1(y)\right)\left(t^{-n} \int_{Q^{\prime}} d x\right) \frac{d y d t}{t} \\
\approx \int_{0}^{\ell(Q)} \int_{Q}\left|\Theta_{t} 1(y)\right|^{2} \mathbb{1}_{\Gamma^{\epsilon}}\left(\Theta_{t} 1(y)\right) \frac{d y d t}{t}
\end{gathered}
$$

Since the latter bound holds for any cone $\Gamma^{\epsilon}$ of aperture $\epsilon$, we may sum over a collection of cones $\left\{\Gamma_{k}^{\epsilon}\right\}$ covering $\mathbb{C}^{m}$, to obtain (3.22).

Thus, it remains only to prove (3.33). We fix a cube Q, and let $\Omega_{N, M}$ be the set defined in 3.31. Our first task is to construct a family of stopping time cubes covering $\Omega_{N, M}$, modulo a set of measure zero. We proceed as follows. Set $F_{N, M}:=Q \backslash \Omega_{N, M}$, and observe that $F_{N, M}$ has positive measure, by (3.32); thus, in particular, the interior of $Q$ (which we denote $\operatorname{int}(Q)$ ), meets $F_{N, M}$. We sub-divide $Q$ dyadically, stopping the first time that we obtain a cube whose interior misses $F_{N, M}$. In this way, we obtain a family $\left\{Q_{j}\right\}$ of dyadic sub-cubes of $Q$, which are maximal with respect to containment of $\operatorname{int}\left(Q_{j}\right)$ in $\Omega_{N, M}$. Obviously, $\cup_{j} \operatorname{int}\left(Q_{j}\right) \subset \Omega_{N, M}$, and we wish to establish the opposite containment, up to a

\footnotetext{
${ }^{2}$ We cannot just use a standard Whitney covering: since we are working with dyadic cones in the definition of $G_{Q, M}$, it is unclear whether the set $\Omega_{N, M}$ is open.

${ }^{3}$ Not to be confused with the family of cubes in the statement of Lemma 3.8 The present cubes have nothing to do with those.
} 
set of measure zero. More precisely, let $Z$ denote the set of measure zero consisting of the union of the boundaries of all cubes in $\mathbb{D}(Q)$. We claim that

$$
\left(\Omega_{N, M} \backslash Z\right) \subset \cup_{j} \operatorname{int}\left(Q_{j}\right) .
$$

Suppose not: then there is a point $x_{0} \in \Omega_{N, M}$ which does not belong to the interior of any $Q_{j}$, nor to the boundary of any dyadic cube. Thus, the interior of every $Q^{\prime} \in \mathbb{D}(Q)$, which contains $x_{0}$, must meet $F_{N, M}$ (otherwise, one such cube would have been a selected $Q_{j}$ ). Let $Q_{0}$ be the unique cube in $\mathbb{D}(Q)$, with side length $2^{-M-1}$, which contains $x_{0}$, so that there is a point $x_{1}$ in $F_{N, M} \cap \operatorname{int}\left(Q_{0}\right)$. Notice that for $Q^{\prime} \in \mathbb{D}_{M}(Q)$, we have that $x_{0} \in Q^{\prime}$ if and only if $x_{1} \in Q^{\prime}$, since in either case, we must have $Q_{0} \subset Q^{\prime}$. Consequently, $\gamma_{Q, M}^{\text {dyad }}\left(x_{0}\right)=\gamma_{Q, M}^{\text {dyad }}\left(x_{1}\right)$, and therefore $G_{Q, M}\left(x_{0}\right)=G_{Q, M}\left(x_{1}\right)$, which contradicts that $x_{0} \in \Omega_{N, M}$ and $x_{1} \in Q \backslash \Omega_{N, M}$. We have therefore shown that

$$
\Omega_{N, M}=\bigcup_{j} Q_{j},
$$

up to a set of measure zero, so by (3.32), we have

$$
\sum_{j}\left|Q_{j}\right| \leq(1-\beta)|Q|
$$

With this covering in hand, we proceed to the proof of (3.33). For convenience of notation, we let $\widetilde{\mathbb{D}}_{M}(Q)$ denote the collection of all $Q^{\prime} \in \mathbb{D}_{M}(Q)$ that are not contained in any $Q_{j}$, and given $x \in Q$, we set

$$
\mathbb{D}_{M}(Q, x):=\left\{Q^{\prime} \in \mathbb{D}_{M}(Q): x \in Q^{\prime}\right\}, \quad \widetilde{\mathbb{D}}_{M}(Q, x):=\left\{Q^{\prime} \in \widetilde{\mathbb{D}}_{M}(Q): x \in Q^{\prime}\right\} .
$$

Recalling the definition of the dyadic cones $\gamma_{Q, M}^{\text {dyad }}$, we observe that for $x \in Q_{j}$, we have

$$
\begin{array}{r}
\gamma_{Q, M}^{\mathrm{dyad}}(x):=\bigcup_{Q^{\prime} \in \mathbb{D}_{M}(Q, x)} U_{Q^{\prime}}=\left(\bigcup_{Q^{\prime} \in \mathbb{D}_{M}\left(Q_{j}, x\right)} U_{Q^{\prime}}\right) \bigcup\left(\bigcup_{Q^{\prime} \in \widetilde{\mathbb{D}}_{M}(Q, x)} U_{Q^{\prime}}\right) \\
=: \gamma_{Q_{j}, M}^{\mathrm{dyad}}(x) \bigcup \tilde{\gamma}_{Q, M}^{\mathrm{dyad}}(x) .
\end{array}
$$

We then make the corresponding definitions

$$
G_{Q_{j}, M}(x):=\left(\iint_{\gamma_{Q_{j}, M}^{\mathrm{dyad}}(x)}\left|\Theta_{t} 1(y)\right|^{2} \mathbb{1}_{\Gamma^{\epsilon}}\left(\Theta_{t} 1(y)\right) \frac{d y d t}{t^{n+1}}\right)^{1 / 2},
$$

and

$$
\widetilde{G}_{Q, M}(x):=\left(\iint_{\tilde{\gamma}_{Q, M}^{\text {dyad }}(x)}\left|\Theta_{t} 1(y)\right|^{2} \mathbb{1}_{\Gamma^{\epsilon}}\left(\Theta_{t} 1(y)\right) \frac{d y d t}{t^{n+1}}\right)^{1 / 2} .
$$

Then by definition, for $x \in Q_{j}$, we have

$$
\left(G_{Q, M}(x)\right)^{2}=\left(G_{Q_{j}, M}(x)\right)^{2}+\left(\widetilde{G}_{Q, M}(x)\right)^{2},
$$

and also, by the stopping time construction,

$$
\widetilde{G}_{Q, M}(x)=\widetilde{G}_{Q, M}\left(x_{1}\right) \leq G_{Q, M}\left(x_{1}\right) \leq N,
$$


for some $x_{1} \in Q \backslash \Omega_{N, M}$. Consequently,

$$
\begin{gathered}
\int_{Q}\left(G_{Q, M}(x)\right)^{2} d x=\int_{F_{N, M}}\left(G_{Q, M}(x)\right)^{2} d x+\sum_{j} \int_{Q_{j}}\left(G_{Q, M}(x)\right)^{2} d x \\
\leq N^{2}|Q|+\sum_{j} \int_{Q_{j}}\left(G_{Q_{j}, M}(x)\right)^{2} d x+\sum_{j} \int_{Q_{j}}\left(\widetilde{G}_{Q, M}(x)\right)^{2} \\
\leq N^{2}|Q|+K(M) \sum_{j}\left|Q_{j}\right|+N^{2} \sum_{j}\left|Q_{j}\right| \\
\leq 2 N^{2}|Q|+K(M)(1-\beta)|Q|,
\end{gathered}
$$

where in the last two inequalities we have used the definition of $K(M)$ and $(3.35)$, and then (3.34). Dividing by $|Q|$, and then taking the supremum over all dyadic $Q$, we obtain

$$
K(M) \leq 2 N^{2}+K(M)(1-\beta) \Longrightarrow K(M) \leq 2 N^{2} / \beta .
$$

\section{Local Tв Theorem Without POINTWISE KERNEL BOUNDS, VERSION 2}

In this section, we present a version of the local $T b$ Theorem for square functions, which may be applied directly to solve the Kato square root problem. We continue to use the notation introduce in the previous section, but we shall modify our hypotheses on the operators $\Theta_{t}$.

Definition 4.1. We consider a family of operators $\left\{\Theta_{t}\right\}_{t>0}$, taking values in $\mathbb{C}^{m}, m \geq 1$, so that $\Theta_{t}:=\left(\Theta_{t}^{1}, \Theta_{t}^{2}, \ldots, \Theta_{t}^{m}\right)$, and for $f=\left(f^{1}, f^{2}, \ldots, f^{m}\right) \in L^{2}\left(\mathbb{R}^{n}, \mathbb{C}^{m}\right)$, we set

$$
\Theta_{t} f:=\sum_{j=1}^{m} \Theta_{t}^{j} f^{j} .
$$

We also define the action of $\Theta_{t}$ on an $m \times m$ matrix valued function $b=\left(b_{j, k}\right)$, in the obvious way, i.e., $\Theta_{t} b=\left(\left(\Theta_{t} b\right)_{1},\left(\Theta_{t} b\right)_{2}, \ldots,\left(\Theta_{t} b\right)_{m}\right)$ is a $\mathbb{C}^{m}$ valued function, with

$$
\left(\Theta_{t} b\right)_{k}:=\sum_{j=1}^{m} \Theta_{t}^{j} b_{j, k} .
$$

We suppose that $\Theta_{t}$ satisfies the following properties:

(a) (Uniform $L^{2}$ bounds and off-diagonal decay in $L^{2}$ ).

$$
\begin{aligned}
& \sup _{t>0}\left\|\Theta_{t} f\right\|_{L^{2}\left(\mathbb{R}^{n}\right)} \leq C\|f\|_{L^{2}\left(\mathbb{R}^{n}\right)}, \\
& \left\|\Theta_{t} f_{j}\right\|_{L^{2}(Q)} \leq C 2^{-j(n+2+\beta) / 2}\left\|f_{j}\right\|_{L^{2}\left(2^{j+1} Q \backslash 2^{j} Q\right)}, \quad \ell(Q) \leq t \leq 2 \ell(Q),
\end{aligned}
$$

for some $\beta>0$, where $f_{j}:=f \mathbb{1}_{2^{j+1} Q \backslash 2^{j} Q}$.

(b) (Quasi-orthogonality on a subspace of $L^{2}$ ). There exists a subspace $H$ of $L^{2}\left(\mathbb{R}^{n}, \mathbb{C}^{m}\right)$, such that for some (hence every) CLP family $\left\{Q_{s}\right\}$ (cf. Definition 3.1), there is a $\beta>0$ for which we have

$$
\left\|\Theta_{t} Q_{s} h\right\|_{L^{2}\left(\mathbb{R}^{n}\right)} \leq C\left(\frac{s}{t}\right)^{\beta}\|h\|_{L^{2}\left(\mathbb{R}^{n}\right)}, \quad \forall h \in H, \quad \forall s \leq t .
$$


Remark 4.1. Let us note that it is only in the quasi-orthogonality condition (b), that we restrict to a sub-space $H \subset L^{2}\left(\mathbb{R}^{n}, \mathbb{C}^{m}\right)$; in condition (a), we suppose that the operator is allowed to act on general $C^{m}$-valued functions in $L^{2}$.

Definition 4.5. Given a subspace $H \subset L^{2}\left(\mathbb{R}^{n}, \mathbb{C}^{m}\right)$, we let $H^{m}$ denote the subspace of $L^{2}\left(\mathbb{R}^{n}, \mathbb{M}^{m}\right)$ (i.e., complex $m \times m$ matrix-valued $L^{2}$ ), consisting of $L^{2}$ matrix-valued functions $b=\left(b_{j, k}\right)$ for which each column vector $b^{k}:=\left(b_{1, k}, b_{2, k}, \ldots, b_{m, k}\right)$ belongs to $H$.

Our main result in this section is the following:

Theorem 4.2. Let $\left\{\Theta_{t}\right\}_{t>0}$, and the subspace $H \subset L^{2}\left(\mathbb{R}^{n}, \mathbb{C}^{m}\right)$, be as in Definition 4.1 above, and suppose that there exist positive constants $C_{0}<\infty$, and $\delta>0$, and a system $\left\{b_{Q}\right\} \subset H^{m}$, indexed by dyadic cubes $Q \subset \mathbb{R}^{n}$, such that for each dyadic cube $Q$ :

$$
\begin{gathered}
\int_{\mathbb{R}^{n}}\left|b_{Q}(x)\right|^{2} d x \leq C_{0}|Q|, \\
\int_{0}^{\ell(Q)} \int_{Q}\left|\Theta_{t} b_{Q}(x)\right|^{2} \frac{d x d t}{t} \leq C_{0}|Q|, \\
\delta|\xi|^{2} \leq \operatorname{Re}\left(\xi \cdot f_{Q} b_{Q}(x) d x \bar{\xi}\right), \quad \forall \xi \in \mathbb{C}^{m} .
\end{gathered}
$$

Then

$$
\iint_{\mathbb{R}_{+}^{n+1}}\left|\Theta_{t} f(x)\right|^{2} \frac{d x d t}{t} \leq C\|f\|_{L^{2}\left(\mathbb{R}^{n}\right)}^{2}, \quad \forall f \in H
$$

The proof of Theorem 4.2 will be based upon the following generalization of the ChristJourné $T 1$ theorem for square functions.

Theorem 4.3. (T1 Theorem) Suppose that $\Theta_{t}$ satisfies conditions (4.2), (4.3) and (4.4) as above, and also that we have the Carleson measure estimate

$$
\sup _{Q} \frac{1}{|Q|} \int_{Q} \int_{0}^{\ell(Q)}\left|\Theta_{t} 1(x)\right|^{2} \frac{d x d t}{t} \leq C .
$$

Then we have the square function estimate

$$
\iint_{\mathbb{R}_{+}^{n+1}}\left|\Theta_{t} f(x)\right|^{2} \frac{d x d t}{t} \leq C\|f\|_{L^{2}\left(\mathbb{R}^{n}\right)}^{2}, \quad \forall f \in H
$$

As above, we view " 1 " in this context as the $m \times m$ identity matrix. We defer the proof of Theorem 4.3 to Section 8 .

Proof of Theorem 4.2 By Theorem 4.3, it suffices to establish 4.10). As in the previous section, we choose an $\epsilon>0$ small enough that Lemma 2.6 holds, and we cover $\mathbb{C}^{m}$ by a family of cones $\left\{\Gamma_{k}^{\epsilon}\right\}_{1 \leq k \leq K(\epsilon, M)}$, of aperture $\epsilon$. It suffices to establish the uniform estimate

$$
\sup _{Q} \frac{1}{|Q|} \int_{0}^{\ell(Q)} \int_{Q}\left|\Theta_{t} 1(x)\right|^{2} \mathbb{1}_{\Gamma^{\epsilon}}\left(\Theta_{t} 1(x)\right) \frac{d x d t}{t} \leq C,
$$

for any fixed cone $\Gamma^{\epsilon}$, since in that case we may apply the latter estimate to each of the cones $\Gamma_{k}^{\epsilon}$, and then sum in $k$ to obtain (4.10). 
Let us then fix a cube $Q$ and a cone $\Gamma^{\epsilon}$. We invoke Lemma 2.6 to obtain a constant $\eta>0$, and a family of non-overlapping cubes $\left\{Q_{j}\right\} \subset \mathbb{D}(Q)$ satisfying

$$
\sum_{j}\left|Q_{j}\right| \leq(1-\eta)|Q|
$$

and

$$
\left|\Theta_{t} 1(x)\right|^{2} \mathbb{1}_{\Gamma^{\epsilon}}\left(\Theta_{t} 1(x)\right) \leq 4\left|\Theta_{t} 1(x) A_{t} b_{Q}(x)\right|^{2}, \quad \forall(x, t) \in E_{Q}^{*}:=R_{Q} \backslash\left(\bigcup_{j} R_{Q_{j}}\right),
$$

where we recall that for any cube $Q, R_{Q}:=Q \times(0, \ell(Q))$ is the corresponding Carleson box. Now, by a well-known John-Nirenberg type lemma for Carleson measures (see, e.g., [H1, Lemma 1.37]), the left hand side of (4.12) is bounded by a constant times

$$
\sup _{Q} \frac{1}{|Q|} \iint_{E_{Q}^{*}}\left|\Theta_{t} 1(x)\right|^{2} \mathbb{1}_{\Gamma^{\epsilon}}\left(\Theta_{t} 1(x)\right) \frac{d x d t}{t} .
$$

In turn, by (4.14), we have

$$
\iint_{E_{Q}^{*}}\left|\Theta_{t} 1(x)\right|^{2} \mathbb{1}_{\Gamma^{\epsilon}}\left(\Theta_{t} 1(x)\right) \frac{d x d t}{t} \leq 4 \iint_{R_{Q}}\left|\Theta_{t} 1(x) A_{t} b_{Q}(x)\right|^{2} \frac{d x d t}{t},
$$

so it suffices to show that the last expression is no larger than a uniform constant time $|Q|$. To this end, as usual we write

$$
\begin{aligned}
\Theta_{t} 1 A_{t} b_{Q}=\left(\left(\Theta_{t} 1\right) A_{t}-\left(\Theta_{t} 1\right) A_{t} P_{t}\right) b_{Q}+\left(\left(\Theta_{t} 1\right) A_{t} P_{t}-\Theta_{t}\right) & b_{Q}+\Theta_{t} b_{Q} \\
= & : R_{t}^{(1)} b_{Q}+R_{t}^{(2)} b_{Q}+\Theta_{t} b_{Q},
\end{aligned}
$$

where as above $P_{t}$ denotes a nice approximate identity operator (cf. Definition 1.3 ). By (4.7), the contribution of $\Theta_{t} b_{Q}$ immediately satisfies the desired bound. We may handle the contributions of the two "remainder" terms $R_{t}^{(1)} b_{Q}$ and $R_{t}^{(2)} b_{Q}$, by invoking (4.6) and Corollary 3.12, which in the present setting is applied only to $h \in H$ (recall that, in particular, each column vector of $b_{Q}$ belongs to $H$, by hypothesis).

\section{Application to the Kato square root problem}

Let $\mathrm{A}$ be an $n \times n$ matrix of complex, $L^{\infty}$ coefficients, defined on $\mathbb{R}^{n}$, and satisfying the ellipticity (or "accretivity") condition

$$
\lambda|\xi|^{2} \leq \mathcal{R} e<A \xi, \xi>\equiv \sum_{i, j} A_{i j}(x) \xi_{j} \bar{\xi}_{i},\|A\|_{\infty} \leq \Lambda,
$$

for $\xi \in \mathbb{C}^{n}$ and for some $\lambda, \Lambda$ such that $0<\lambda \leq \Lambda<\infty$. We define the divergence form operator

$$
L u:=-\operatorname{div}(A(x) \nabla u),
$$

which we interpret in the usual weak sense via a sesquilinear form.

The accretivity condition above enables one to define an accretive square root $\sqrt{L}$.

Theorem 5.1. [AHLMcT] Let $L$ be a divergence form operator defined as above. Then for all $h \in \dot{L}_{1}^{2}\left(\mathbb{R}^{n}\right)$, we have

$$
\|\sqrt{L} h\|_{L^{2}\left(\mathbb{R}^{n}\right)} \leq C\|\nabla h\|_{L^{2}\left(\mathbb{R}^{n}\right)},
$$

with $C$ depending only on $n, \lambda$ and $\Lambda$. 
Proof. In [AT], it is shown that the conclusion of Theorem[5.1] is equivalent to the square function estimate

$$
\iint_{\mathbb{R}_{+}^{n+1}}\left|\Theta_{t} \nabla h\right|^{2} \frac{d x d t}{t} \leq C_{n, \lambda, \Lambda} \int_{\mathbb{R}^{n}}|\nabla h|^{2} d x,
$$

where $\Theta_{t}:=t e^{-t^{2} L} \operatorname{div} A$. Thus, to prove this theorem, it is enough to verify the conditions of the Local Tb Theorem (Theorem 4.2), for this operator $\Theta_{t}$, with $m=n$, and with

$$
H:=\left\{\nabla h: h \in \dot{L}_{1}^{2}\left(\mathbb{R}^{n}, \mathbb{C}^{n}\right)\right\},
$$

a subspace of $L^{2}\left(\mathbb{R}^{n}, \mathbb{C}^{n}\right)$.

(a) It follows from standard semigroup theory for divergence form elliptic operators that the family $\left\{t e^{-t^{2} L} \mathrm{div}\right\}_{t>0}$ satisfies uniform $L^{2}$ bounds, as well as $L^{2}$ off-diagonal estimates (for the latter, just dualize the well known "Gaffney estimates" for $t \nabla e^{-t^{2} L}$ ). Thus, since $A \in L^{\infty}\left(\mathbb{R}^{n}\right)$, we have condition (a) for $\Theta_{t}$.

(b) We choose $\left\{Q_{s}\right\}_{s>0}$ of convolution type satisfying the required conditions of Definition 3.1. and in addition we choose the kernel $\psi \in C_{0}^{\infty}$. We note that $Q_{s} \nabla F=\nabla Q_{s} F$. Let $\nabla F \in H$, where (by definition) $F \in \dot{L}_{1}^{2}\left(\mathbb{R}^{n}\right)$. Then $F$ is equivalent (modulo constants) to the realization $F=I_{1} f$, where $f \in L^{2}\left(\mathbb{R}^{n}\right)$, with $\|f\|_{2} \approx\|\nabla F\|_{2}$, and $I_{1}$ is the Riesz potential with $\alpha=1$ as in Definition 1.5 . We then have

$$
\begin{aligned}
t e^{-t^{2} L} \operatorname{div} A Q_{s} \nabla F & =t e^{-t^{2} L} \operatorname{div} A \nabla Q_{s} F \\
& =-t L e^{-t^{2} L} Q_{s} F \\
& =-\frac{1}{t} t^{2} L e^{-t^{2} L} Q_{s} I_{1} f \\
& =\frac{-s}{t} t^{2} L e^{-t^{2} L}\left(\frac{1}{s} Q_{s} I_{1}\right) f .
\end{aligned}
$$

Using the fact that $t^{2} L e^{-t^{2} L}: L^{2} \rightarrow L^{2}$ and $\frac{1}{s} Q_{s} I_{1}: L^{2} \rightarrow L^{2}$ we obtain condition (b).

Finally, we need to find a family of $b_{Q}$ indexed by cubes $\mathrm{Q}$ satisfying the required

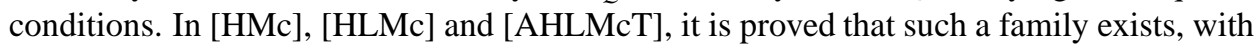
$b_{Q}$ of the form

$$
b_{Q}=\nabla F_{Q}:=e^{-\varepsilon \ell(Q)^{2} L}\left(\varphi_{Q}\right),
$$

with $\varphi_{Q}(x):=\left(x-x_{Q}\right) \eta_{Q}$, where $\varepsilon>0$, is a number chosen suitably small depending only on $n, \lambda$ and $\Lambda$, and where $x_{Q}$ denotes the center of $Q$, and $\eta_{Q}$ is a smooth cut-off function which is 1 on $4 Q$ and vanishes outside of $5 Q$. We refer the reader to [AHLMcT] for details of the proofs of the required properties.

\section{A local $T b$ THEOREM With VECTOR-VALUED TESTING FUNCTIONS}

In this section, we consider a version of the local $T b$ theorem for vector-valued $\Theta_{t}$, in which the testing functions themselves are vector-valued rather than matrix-valued. In the following section, we shall then apply this version to establish $L^{2}$ bounds for layer potentials associated to a class of divergence form elliptic operators in the half-space $\mathbb{R}_{+}^{n+1}$.

Definition 6.1. We consider a family of operators $\left\{\Theta_{t}\right\}_{t>0}$, taking values in $\mathbb{C}^{m+1}, m \geq 1$, so that $\Theta_{t}:=\left(\Theta_{t}^{0}, \Theta_{t}^{\prime}\right):=\left(\Theta_{t}^{0}, \Theta_{t}^{1}, \Theta_{t}^{2}, \ldots, \Theta_{t}^{m}\right)$, where each $\Theta_{t}^{j}, 0 \leq j \leq m$, acts on scalar 
valued $L^{2}$, and where for $g=\left(g^{0}, g^{\prime}\right):=\left(g^{0}, g^{1}, \ldots, g^{m}\right) \in L^{2}\left(\mathbb{R}^{n}, \mathbb{C}^{m+1}\right)$, we set

$$
\Theta_{t} g=\sum_{j=0}^{m} \Theta_{t}^{j} g^{j}, \quad \Theta_{t}^{\prime} g^{\prime}=\sum_{j=1}^{m} \Theta_{t}^{j} g^{j}
$$

We suppose that $\Theta_{t}$ satisfies the following properties:

(a) (Uniform $L^{2}$ bounds and off-diagonal decay in $L^{2}$ ).

$$
\begin{gathered}
\sup _{t>0}\left\|\Theta_{t} g\right\|_{L^{2}\left(\mathbb{R}^{n}\right)} \leq C\|g\|_{L^{2}\left(\mathbb{R}^{n}, \mathbb{C}^{m}\right)}, \\
\left\|\Theta_{t} g_{j}\right\|_{L^{2}(Q)} \leq C 2^{-j(n+2+\beta) / 2}\left\|g_{j}\right\|_{L^{2}\left(2^{j+1} Q \backslash 2^{j} Q\right)}, \quad \ell(Q) \leq t \leq 2 \ell(Q),
\end{gathered}
$$

for some $\beta>0$, where $g_{j}:=g \mathbb{1}_{2^{j+1} Q \backslash 2^{j} Q}$;

(b) (Quasi-orthogonality on a subspace of $L^{2}$ ). There exists a subspace $H$ of $L^{2}\left(\mathbb{R}^{n}, \mathbb{C}^{m}\right)$, such that for some (hence every) CLP family $\left\{Q_{s}\right\}$ (cf. Definition 3.1 ), there is a $\beta>0$ for which we have

$$
\left\|\Theta_{t} Q_{s} h\right\|_{L^{2}\left(\mathbb{R}^{n}\right)} \leq C\left(\frac{s}{t}\right)^{\beta}\|h\|_{L^{2}\left(\mathbb{R}^{n}\right)}, \quad \forall h=\left(h^{0}, h^{\prime}\right) \in L^{2}\left(\mathbb{R}^{n}, \mathbb{C}\right) \times H, \forall s \leq t .
$$

We further define the action of $\Theta_{t}$ (respectively, $\left.\Theta_{t}^{\prime}\right)$ on matrix-valued $B=\left(B_{i j}\right)_{0 \leq i, j \leq m}$ : $\mathbb{R}^{n} \rightarrow \mathbb{M}^{m+1}$ (resp., $B^{\prime}=\left(B_{i j}\right)_{1 \leq i, j \leq m}: \mathbb{R}^{n} \rightarrow \mathbb{M}^{m}$ ) in the obvious way:

$$
\Theta_{t} B(x)=\left(\sum_{i=0}^{m} \Theta_{t}^{i}(x, y) B_{i j}\right)_{0 \leq j \leq m} \Theta_{t}^{\prime} B^{\prime}(x)=\left(\sum_{i=1}^{m} \Theta_{t}^{i}(x, y) B_{i j}\right)_{1 \leq j \leq m} .
$$

For $\Theta_{t}$ as above, for convenience of notation, we set

$$
\zeta(x, t):=\Theta_{t} 1(x), \quad \zeta^{\prime}(x, t):=\Theta_{t}^{\prime} 1(x), \quad \zeta^{0}(x, t):=\Theta_{t}^{0} 1(x),
$$

where in the previous line, " 1 " denotes, respectively, the $(m+1) \times(m+1)$ identity matrix. the $m \times m$ identity matrix, and the scalar constant 1 . For $\delta \in[0,1)$, we also set

$$
\zeta_{\delta}(x, t):=\zeta(x, t) 1_{\{\delta<t<1 / \delta\}}, \quad \zeta_{\delta}^{\prime}(x, t):=\zeta^{\prime}(x, t) 1_{\{\delta<t<1 / \delta\}}, \quad \zeta_{\delta}^{0}(x, t):=\zeta^{0}(x, t) 1_{\{\delta<t<1 / \delta\}} .
$$

Definition 6.6. Given $\Upsilon$ (either scalar or vector valued), defined on $\mathbb{R}_{+}^{n+1}$, we set

$$
\|\Upsilon\|_{C}:=\sup _{Q} \frac{1}{|Q|} \int_{0}^{\ell(Q)} \int_{Q}|\Upsilon(x, t)|^{2} \frac{d x d t}{t} .
$$

Definition 6.7. Given a dyadic cube $Q$, and a positive constant $C_{0}$, we shall say that a Borel measure $\mu_{Q}$ on $\mathbb{R}^{n}$ is "adapted to $\mathrm{Q}$ with constant $C_{0}$ ", if

$$
d \mu_{Q}=\phi_{Q} d x
$$

where $\phi_{Q}: \mathbb{R}^{n} \rightarrow[0,1]$ is a Lipschitz function satisfying

$$
\left\|\nabla \phi_{Q}\right\|_{L^{\infty}\left(\mathbb{R}^{n}\right)} \leq C_{0} \ell(Q)^{-1},
$$

and also

$$
\frac{1}{C_{0}} \leq \phi_{Q}, \quad \text { on } Q
$$

We observe that $\phi_{Q}$, being Lipschitz, is not supported in $Q$, by 6.9. 
Theorem 6.1. Let $\Theta_{t}$ and $H$ be as in Definition 6.1 and suppose that there exist positive constants $\sigma, \eta, C_{0}$ and $C_{1}$, a system $\left\{b_{Q}\right\}=\left\{\left(b_{Q}^{0}, b_{Q}^{\prime}\right)\right\} \subset L^{2}\left(\mathbb{R}^{n}, \mathbb{C}\right) \times H \subset L^{2}\left(\mathbb{R}^{n}, \mathbb{C}^{m+1}\right)$, indexed by dyadic cubes $Q \subset \mathbb{R}^{n}$, and a system of measures $\left\{\mu_{Q}\right\}$, also indexed by dyadic cubes, with each $\mu_{Q}$ "adapted to $Q$ with constant $C_{0}$ " as in Definition 6.7 such that for every dyadic cube $Q$, we have

$$
\begin{gathered}
\int_{\mathbb{R}^{n}}\left|b_{Q}(x)\right|^{2} d x \leq C_{0}|Q|, \\
\int_{0}^{\ell(Q)} \int_{Q}\left|\Theta_{t} b_{Q}(x)\right|^{2} \frac{d x d t}{t} \leq C_{0}|Q|, \\
\sigma \leq \operatorname{Re}\left(f_{Q} b_{Q}^{0} d \mu_{Q}\right), \\
\left|f_{Q} b_{Q}^{\prime} d \mu_{Q}\right| \leq \eta \sigma,
\end{gathered}
$$

with $\eta \leq 1 /\left(2 C_{1}+4\right)$, where, for all sufficiently small $\delta>0$ (hence also for $\delta=0$ ),

$$
\left\|\zeta_{\delta}^{\prime}\right\|_{C} \leq C_{1}\left(1+\left\|\zeta_{\delta}^{0}\right\|_{C}\right)
$$

Then for all $f^{0} \in L^{2}\left(\mathbb{R}^{n}, \mathbb{C}\right)$,

$$
\iint_{\mathbb{R}_{+}^{n+1}}\left|\Theta_{t}^{0} f^{0}(x)\right|^{2} \frac{d x d t}{t} \leq C\left\|f^{0}\right\|_{2}^{2}
$$

Remark 6.2. Given the hypotheses of Theorem 6.1, we also have that for all $h^{\prime} \in H$,

$$
\iint_{\mathbb{R}_{+}^{n+1}}\left|\Theta_{t}^{\prime} h^{\prime}(x)\right|^{2} \frac{d x d t}{t} \leq C\left\|h^{\prime}\right\|_{2}^{2}
$$

Indeed, by a well known argument of [FS], 6.15) implies that $\left\|\Theta_{t}^{0} 1\right\|_{C} \leq C$, whence (6.16) follows immediately by (6.14) and Theorem 4.3

Remark 6.3. It may be that in some applications, one could simply take $\phi_{Q} \equiv 1$, i.e., $d \mu_{Q}=d x$, but in our application in Section 7 it is useful to have the extra flexibility inherent in 6.12)- 6.13).

Remark 6.4. As a practical matter, in applications, one expects to construct $b_{Q}^{\prime}$ depending on $\eta$, where the parameter $\eta$ in (6.13) is at one's disposal and can therefore be made sufficiently small. This is precisely what we shall do in applying Theorem 6.1 to the study of variable coefficient layer potentials, in Section 7 We emphasize that $\eta$ is required to be small depending only upon $C_{1}$, but not on $C_{0}$; thus, in applications, there is no harm if $C_{0}$ becomes larger as $\eta$ becomes smaller, as indeed, will be the case in our application in Section 7

Proof. By Theorem 3.6 (the generalized $T 1$ Theorem of $[\overline{\mathrm{CJ}}]$ ), it is enough to show that $\left\|\zeta^{0}\right\|_{C} \leq C$. We proceed under the a priori qualitative assumption that $\left\|\zeta^{0}\right\|_{C}$ is finite, but with no particular quantitative bound. We may do this by working with $\zeta_{\delta}$ in place of $\zeta$, as long as we obtain bounds that are independent of $\delta$. To simplify the notation, we shall simply write $\zeta$, not $\zeta_{\delta}$. We define

$$
\begin{aligned}
& F_{1}:=\left\{(x, t) \in \mathbb{R}_{+}^{n+1}:\left|\zeta^{0}(x, t)\right| \leq \sqrt{\eta}\left|\zeta^{\prime}(x, t)\right|\right\} \\
& F_{2}:=\left\{(x, t) \in \mathbb{R}_{+}^{n+1}:\left|\zeta^{0}(x, t)\right|>\sqrt{\eta}\left|\zeta^{\prime}(x, t)\right|\right\},
\end{aligned}
$$

so that

$$
\left\|\zeta^{0}\right\|_{C} \leq\left\|\zeta^{0} 1_{F_{1}}\right\|_{C}+\left\|\zeta^{0} 1_{F_{2}}\right\|_{C}
$$


By Definition 6.6, and 6.14), we have

$$
\left\|\zeta^{0} 1_{F_{1}}\right\|_{C} \leq \eta\left\|\zeta^{\prime}\right\|_{C} \leq C_{1} \eta\left(1+\left\|\zeta^{0}\right\|_{C}\right) .
$$

Since $\eta \leq 1 /\left(2 C_{1}\right)$, we may hide the term $C_{1} \eta\left\|\zeta^{0}\right\|_{C}$ on the left hand side of 6.17). Thus, it suffices to show that $\left\|\zeta^{0} 1_{F_{2}}\right\|_{C} \leq C$. To this end, we first note that by 6.12) and (6.13),

$$
\sigma\left|\zeta^{0}\right| \leq\left|\zeta^{0} f_{Q} b_{Q}^{0} d \mu_{Q}\right| \leq\left|\zeta \cdot f_{Q} b_{Q} d \mu_{Q}\right|+\left|\zeta^{\prime} \cdot f_{Q} b_{Q}^{\prime} d \mu_{Q}\right| \leq\left|\zeta \cdot f_{Q} b_{Q} d \mu_{Q}\right|+\eta \sigma\left|\zeta^{\prime}\right|,
$$

for every dyadic cube $Q$. In $F_{2}$, we then have (again for every $Q$ )

$$
\sigma\left|\zeta^{0}\right| \leq\left|\zeta \cdot f_{Q} b_{Q} d \mu_{Q}\right|+\sqrt{\eta} \sigma\left|\zeta^{0}\right|
$$

and also

$$
|\zeta| \leq\left|\zeta^{0}\right|+\left|\zeta^{\prime}\right| \leq\left(1+\eta^{-1 / 2}\right)\left|\zeta^{0}\right| \leq 2 \eta^{-1 / 2}\left|\zeta^{0}\right| .
$$

Combining the latter two estimates, we obtain that for all $(x, t) \in F_{2}$, and for every $Q$,

$$
\frac{\sqrt{\eta}}{2}(1-\sqrt{\eta}) \sigma|\zeta(x, t)| \leq(1-\sqrt{\eta}) \sigma\left|\zeta^{0}(x, t)\right| \leq\left|\zeta(x, t) \cdot f_{Q} b_{Q} d \mu_{Q}\right| .
$$

We now observe that, as above, in order to estimate $\left\|\zeta^{0} 1_{F_{2}}\right\|_{C}$, it suffices to prove that, for $\epsilon>0$ chosen small enough,

$$
\left\|\zeta^{0} 1_{F_{2}} \mathbb{1}_{\Gamma^{\epsilon}}(\zeta)\right\|_{C} \leq C
$$

where $\Gamma^{\epsilon}$ is an arbitrary cone of aperture $\epsilon$, i.e.,

$$
\Gamma^{\epsilon}=\Gamma^{\epsilon}(v):=\left\{z \in \mathbb{C}^{m+1}:|(z /|z|)-v|<\epsilon\right\},
$$

for some unit direction vector $v \in \mathbb{C}^{m+1}$. Indeed, given 6.19), we may then sum over an appropriate collection of such cones, covering $\mathbb{C}^{m+1}$, to obtain the bound $\left\|\zeta^{0} 1_{F_{2}}\right\|_{C} \leq$ $C(\epsilon, m)$. We therefore fix such a cone $\Gamma^{\epsilon}=\Gamma^{\epsilon}(v)$, and observe that, for $(x, t) \in F_{2}$, and $\zeta(x, t) \in \Gamma^{\epsilon}$, since $\eta \leq 1 / 4$, by 6.18 , we have for every dyadic cube $Q$,

$$
\begin{array}{r}
\frac{\sqrt{\eta}}{4} \sigma \leq\left|\frac{\zeta(x, t)}{|\zeta(x, t)|} \cdot f_{Q} b_{Q} d \mu_{Q}\right| \leq\left|\left(\frac{\zeta(x, t)}{|\zeta(x, t)|}-v\right) \cdot f_{Q} b_{Q} d \mu_{Q}\right|+\left|v \cdot f_{Q} b_{Q} d \mu_{Q}\right| \\
\leq C_{0} \epsilon+\left|v \cdot f_{Q} b_{Q} d \mu_{Q}\right|,
\end{array}
$$

where in the last step we have used Schwarz's inequality, the fact that $1 / C_{0} \leq d \mu_{Q} / d x=$ $\phi_{Q} \leq 1$ on $Q$, and 6.10). Since $\epsilon$ is at our disposal, we may choose it small enough, say $\epsilon \leq \sigma \sqrt{\eta} /\left(8 C_{0}\right)$, and then hide the small term, to obtain

$$
\frac{\sqrt{\eta}}{8} \sigma=: \theta \leq\left|v \cdot f_{Q} b_{Q} d \mu_{Q}\right| \text {. }
$$

We observe that the latter bound does not depend on $(x, t)$, but on the other hand, was deduced from the existence of some $(x, t) \in F_{2}$, for which $\zeta(x, t) \in \Gamma^{\epsilon}$. Thus, (6.20) holds for any $Q$ such that

$$
\iint_{R_{Q}}\left|\zeta^{0}(x, t)\right|^{2} 1_{F_{2}}(x, t) \mathbb{1}_{\Gamma^{\epsilon}}(\zeta(x, t)) \frac{d x d t}{t} \neq 0
$$

where as usual, $R_{Q}:=Q \times(0, \ell(Q))$ denotes the standard Carleson box above $Q$. Consequently, in proving 6.19, we may suppose henceforth, without loss of generality, that 6.20) holds in any dyadic cube $Q$ under consideration. 
We therefore fix a dyadic cube $Q$ such that (6.20) holds, and we follow a (now familiar) stopping time procedure to extract a family $\left\{Q_{j}\right\}$ of non-overlapping dyadic sub-cubes of $Q$, which are maximal with respect to the property that at least one of the following conditions holds:

$$
\begin{gathered}
f_{Q_{j}}\left|b_{Q}\right| d \mu_{Q}>\frac{\theta}{4 \epsilon} \quad \text { (type I) } \\
\left|v \cdot f_{Q_{j}} b_{Q} d \mu_{Q}\right| \leq \frac{\theta}{2} \quad \text { (type II). }
\end{gathered}
$$

If some $Q_{j}$ happens to satisfy both the type I and type II conditions, then we arbitrarily assign it to be of type II; for simplicity of notation, we write $Q_{j} \in I$, or $Q_{j} \in I I$, to mean that the cube is of type I, or type II, respectively. We now claim that for this family of cubes,

$$
\sum_{j}\left|Q_{j}\right| \leq(1-\beta)|Q|
$$

for some uniform $\beta>0$, and that

$$
|\zeta(x, t)|^{2} \mathbb{1}_{\Gamma^{\epsilon}}(\zeta(x, t)) \leq C_{\theta}\left|\zeta(x, t) \cdot A_{t}^{\mu_{Q}} b_{Q}(x)\right|^{2}, \quad \forall(x, t) \in E_{Q}^{*}:=R_{Q} \backslash\left(\bigcup_{j} R_{Q_{j}}\right),
$$

where $A_{t}^{\mu_{Q}}$ is the dyadic averaging operator adapted to the measure $\mu_{Q}$, i.e.,

$$
A_{t}^{\mu_{Q}} f(x):=\int_{Q(x, t)} f d \mu_{Q},
$$

where $Q(x, t)$ denotes the smallest dyadic cube, of side length at least $t$, that contains $x$.

Let us verify 6.24) first. Observe first that for any dyadic sub-cube $Q^{\prime} \subset Q$, which is not contained in any $Q_{j}$, we have that the opposite inequalities to (6.21) and 6.22) hold, for the average of $b_{Q}$ over $Q^{\prime}$, by maximality of the cubes in the family $\left\{Q_{j}\right\}$; i.e., by definition of $A_{t}^{\mu_{Q}}$, we have

$$
\frac{\theta}{2} \leq\left|v \cdot A_{t}^{\mu_{Q}}\left(b_{Q}\right)(x)\right| \text { and }\left|A_{t}^{\mu_{Q}}\left(b_{Q}\right)(x)\right| \leq \frac{\theta}{4 \epsilon}, \quad \forall(x, t) \in E_{Q}^{*} .
$$

Thus, if $z \in \Gamma^{\epsilon}$, and $(x, t) \in E_{Q}^{*}$, we have

$$
\begin{aligned}
\frac{\theta}{2} \leq\left|v \cdot A_{t}^{\mu_{Q}}\left(b_{Q}\right)(x)\right| \leq\left|(z /|z|) \cdot A_{t}^{\mu_{Q}}\left(b_{Q}\right)(x)\right|+\left|(v-(z /|z|)) \cdot A_{t}^{\mu_{Q}}\left(b_{Q}\right)(x)\right| & \leq\left|(z /|z|) \cdot A_{t}^{\mu_{Q}}\left(b_{Q}\right)(x)\right|+\frac{\theta}{4} .
\end{aligned}
$$

We now obtain 6.24), by setting $z=\zeta(x, t)$.

Next, we establish 6.23). Set $E:=Q \backslash\left(\cup_{j} Q_{j}\right)$, and $B_{1}:=\cup_{Q_{j} \in I} Q_{j}$. We note that by definition of the type I cubes, $B_{1} \subset\left\{M\left(b_{Q}\right)>\theta /(4 \epsilon)\right\}$, where $M$ denotes the HardyLittlewood maximal operator, whence it follows by (6.10) that,

$$
\left|B_{1}\right| \leq C\left(\frac{\epsilon}{\theta}\right)^{2} \int_{\mathbb{R}^{n}}\left|b_{Q}\right|^{2} \leq C C_{0}\left(\frac{\epsilon}{\theta}\right)^{2}|Q| .
$$

Let us note that by Definition 6.7

$$
\frac{1}{C_{0}}|Q| \leq \mu_{Q}(Q) \leq|Q| .
$$


By (6.20), and then Definition 6.7 6.22, 6.25), and 6.10), we have

$$
\begin{aligned}
& \theta \mu_{Q}(Q) \leq\left|v \cdot \int_{Q} b_{Q} d \mu_{Q}\right| \\
& \quad \leq\left|v \cdot \int_{E} b_{Q} d \mu_{Q}\right|+\int_{B_{1}}\left|b_{Q}\right| d \mu_{Q}+\sum_{Q_{j} \in I I}\left|v \cdot \int_{Q_{j}} b_{Q} d \mu_{Q}\right| \\
& \leq|E|^{1 / 2}\left\|b_{Q}\right\|_{2}+\left|B_{1}\right|^{1 / 2}\left\|b_{Q}\right\|_{2}+\frac{\theta}{2} \sum_{j} \mu_{Q}\left(Q_{j}\right) \leq C|E|^{1 / 2}|Q|^{1 / 2}+C_{\theta} \epsilon|Q|+\frac{\theta}{2} \mu(Q) .
\end{aligned}
$$

Choosing $\epsilon$ small enough, and using (6.26), we have $|Q| \leq C_{\theta}|E|$, which is equivalent to 6.23 .

With 6.23) and 6.24) in hand, we turn to the proof of 6.19). We note that by 6.23, and a standard John-Nirenberg lemma for Carleson measures (see, e.g., [H1, Lemma $1.37])$,

$$
\begin{aligned}
&\left\|\zeta^{0} 1_{F_{2}} \mathbb{1}_{\Gamma^{\epsilon}}(\zeta)\right\|_{C} \lesssim \sup _{Q} \frac{1}{|Q|} \iint_{E_{Q}^{*}}\left|\zeta^{0}(x, t)\right|^{2} 1_{F_{2}}(x, t) \mathbb{1}_{\Gamma^{\epsilon}}(\zeta(x, t)) \frac{d x d t}{t} \\
& \lesssim \sup _{Q} \frac{1}{|Q|} \iint_{R_{Q}}\left|\zeta(x, t) \cdot A_{t}^{\mu_{Q}} b_{Q}(x)\right|^{2} \frac{d x d t}{t},
\end{aligned}
$$

where in the last step we have used the trivial bound $\left|\zeta^{0}\right| \leq|\zeta|$, and (6.24).

Thus it remains only to show that the last term is bounded. To this end, recalling that $\zeta=\Theta_{t} 1$, we fix $Q$, and use the familiar trick of Coifman-Meyer to write

$$
\begin{aligned}
\zeta \cdot A_{t}^{\mu_{Q}} b_{Q}=\left(\left(\Theta_{t} 1\right) A_{t}^{\mu_{Q}}-\left(\Theta_{t} 1\right) A_{t}^{\mu_{Q}} P_{t}\right) b_{Q}+\left(\left(\Theta_{t} 1\right) A_{t}^{\mu_{Q}} P_{t}\right. & \left.-\Theta_{t}\right) b_{Q}+\Theta_{t} b_{Q} \\
& =: R_{t}^{(1)} b_{Q}+R_{t}^{(2)} b_{Q}+\Theta_{t} b_{Q},
\end{aligned}
$$

where as above $P_{t}$ denotes a nice approximate identity operator (cf. Definition 1.3). The contribution of $\Theta_{t} b_{Q}$ may be handled immediately by hypothesis (6.11). The contributions of the two remainder terms $R_{t}^{(i)} b_{Q}, i=1,2$, may be handled as follows. By 6.10 , and a standard orthogonality argument, it is enough to show that for some $\beta_{0}>0$, and for all $t \in(0, \ell(Q))$, we have

$$
\int_{Q}\left|R_{t}^{(i)} Q_{s} h(y)\right|^{2} d y \lesssim \min \left(\frac{s}{t}, \frac{t}{s}\right)^{\beta_{0}} \int_{\mathbb{R}^{n}}|h(x)|^{2} d x .
$$

In turn, one may prove the latter fact by following mutatis mutandi the proof of Lemma 3.11 bearing in mind that in $R_{Q}$, by Definition 6.7 the modified dyadic averages $A_{t}^{\mu_{Q}}$ enjoy the same estimates as do the standard dyadic averages $A_{t}$.

\section{ApPlication of Theorem 6.1 to the THEORY OF LAYER POTENTIALS}

We consider layer potentials associated to divergence form complex coefficient equations $L u=0$, where

$$
L=-\operatorname{div} A \nabla:=-\sum_{i, j=1}^{n+1} \frac{\partial}{\partial x_{i}}\left(A_{i, j} \frac{\partial}{\partial x_{j}}\right)
$$

is defined in $\mathbb{R}^{n+1}=\left\{(x, t) \in \mathbb{R}^{n} \times \mathbb{R}\right\}, n \geq 2$ (we recall that we use the notational conventions that $x_{n+1}=t$, and that capital letters may be used to denote points in $\mathbb{R}^{n+1}$, e.g., $X=(x, t)$ ). 
Here, $A=A(x)$ is an $(n+1) \times(n+1)$ matrix of complex-valued $L^{\infty}$ coefficients, defined on $\mathbb{R}^{n}$ (i.e., independent of the $t$ variable) and satisfying the uniform ellipticity condition

$$
\lambda|\xi|^{2} \leq \mathfrak{R} e\langle A(x) \xi, \xi\rangle:=\mathfrak{R} e \sum_{i, j=1}^{n+1} A_{i j}(x) \xi_{j} \bar{\xi}_{i}, \quad\|A\|_{L^{\infty}\left(\mathbb{R}^{n}\right)} \leq \Lambda,
$$

for some $\lambda>0, \Lambda<\infty$, and for all $\xi \in \mathbb{C}^{n+1}, x \in \mathbb{R}^{n}$.

In the present work, we further suppose that weak solutions of the equations $L u=0$ (and also $L^{*} u=0$, where $L^{*}$ denotes the Hermitian adjoint of $L$ ) satisfy De Giorgi/Nash ("DG/N") estimates, i.e., that there is a constant $C$ and an exponent $\alpha>0$ such that for any ball $B=B(X, R) \subset \mathbb{R}^{n+1}$, of radius $R$, for which $L u=0$ in the concentric double $2 B:=B(X, 2 R)$, we have the local Hölder continuity estimate

$$
|u(Y)-u(Z)| \leq C\left(\frac{|Y-Z|}{R}\right)^{\alpha}\left(f_{2 B}|u|^{2}\right)^{1 / 2},
$$

whenever $Y, Z \in B$. Observe that any $u$ satisfying (7.2) also satisfies Moser's "local boundedness" estimate $[\overline{\mathrm{M}}]$

$$
\sup _{Y \in B}|u(Y)| \leq C\left(f_{2 B}|u|^{2}\right)^{1 / 2}
$$

Estimates (7.2) and (7.3) always hold for real coefficients [DeG,, $\mathbf{N}]$, and are stable under small complex, $L^{\infty}$ perturbations [A2] (see also [AT, HK2]); moreover they hold always for $t$-independent complex operators in ambient dimension $n+1=3$ AAAHK, Section 11]. In the presence of the DG/N estimates (for $L$ and $L^{*}$ ), by [ [HK] , the operators $L$ and $L^{*}$, respectively, have fundamental solutions $E(X, Y)$, and $E^{*}(X, Y)=\overline{E(Y, X)}$, satisfying the following properties: first, that

$$
L_{x, t} E(x, t, y, s)=\delta_{(y, s)}, \quad L_{y, s}^{*} E^{*}(y, s, x, t):=L_{y, s}^{*} \overline{E(x, t, y, s)}=\delta_{(x, t)},
$$

where $\delta_{X}$ denotes the Dirac mass at the point $X$; second, by the $t$-independence of our coefficients, that

$$
E(x, t, y, s)=E(x, t-s, y, 0)
$$

and finally, for $j \geq 0$, that there exists a constant $C_{j}$ depending only on $j$, dimension, ellipticity and (7.2) and (7.3), such that for for all $(x, t) \neq(y, s) \in \mathbb{R}^{n+1}$, we have

$$
\begin{gathered}
\left|\left(\partial_{t}\right)^{j} E(x, t, y, s)\right| \leq C_{j}(|t-s|+|x-y|)^{1-n-j} \\
\left|\left(\Delta_{h}\left(\partial_{t}\right)^{j} E(\cdot, t, y, 0)\right)(x)\right|+\left|\left(\Delta_{h}\left(\partial_{t}\right)^{j} E(x, t, \cdot, 0)\right)(y)\right| \leq C_{j} \frac{|h|^{\alpha}}{(|t|+|x-y|)^{n+j+\alpha-1}},
\end{gathered}
$$

whenever $2|h| \leq|x-y|$ or $|h|<20|t|$, for some $\alpha>0$, where $\left(\Delta_{h} f\right)(x):=f(x+h)-f(x)$.

We define the single layer potential operator, associated to $L$, by

$$
\mathcal{S}_{t} f(x):=\int_{\mathbb{R}^{n}} E(x, t, y, 0) f(y) d y, t \in \mathbb{R} ;
$$

the single layer potential associated to $L^{*}$ is defined analogously, with $E^{*}$ in place of $E$.

We shall encounter operators whose kernels involve derivatives applied to the second set of variables in the fundamental solution $E(x, t, y, s)$, and we denote this by appropriate parenthetic grouping; e.g., for $f: \mathbb{R}^{n} \rightarrow \mathbb{C}^{n+1}$, we set

$$
\left(\mathcal{S}_{t} \nabla\right) f(x):=\left.\int_{\mathbb{R}^{n}}\left(\nabla_{y, s} E(x, t, y, s)\right)\right|_{s=0} \cdot f(y) d y .
$$


We similarly denote the individual components of the vector-valued operator $\left(\mathcal{S}_{t} \nabla\right)$, thus for $f: \mathbb{R}^{n} \rightarrow \mathbb{C}^{n}$,

$$
\left(\mathcal{S}_{t} \nabla y\right) f=-\mathcal{S}_{t}\left(\operatorname{div}_{y} f\right)
$$

and, by translation invariance in $t$ (7.5),

$$
\left(\mathcal{S}_{t} \partial_{s}\right)=-\partial_{t} \mathcal{S}_{t}
$$

With these notational conventions in hand, we record for future reference the following estimates taken from [AAAHK]:

Lemma 7.1 ([AAAHK], Lemma 2.8, Lemma 2.9 and Lemma 2.10). Suppose that $L, L^{*}$ are $t$-independent divergence form complex elliptic operators as above, whose null solutions satisfy the DGN bounds (7.2). Then for some C depending only on $n$, (7.1) and (7.2), for every fixed $x \in \mathbb{R}^{n}$ and $t \neq 0$, we have

$$
\left.\int_{\mathbb{R}^{n}}\left|\nabla_{y, s} E(y, s, x, t)\right|_{s=0}\right|^{2} d y \leq C|t|^{-n}
$$

In addition, if $j \geq 0$, then there is a constant $C_{j}$ depending on $n$, (7.1) and (7.2), such that for $f: \mathbb{R}^{n} \rightarrow \mathbb{C}^{n+1}$, and for every cube $Q$, for all integers $k \geq 1$, and for all $t \in \mathbb{R}$,

$$
\left\|\left(\partial_{t}\right)^{j}\left(\mathcal{S}_{t} \nabla\right)\left(f 1_{2^{k+1} Q \backslash 2^{k} Q}\right)\right\|_{L^{2}(Q)}^{2} \leq C_{j} 2^{-n k}\left(2^{k} \ell(Q)\right)^{-2 j}\|f\|_{L^{2}\left(2^{k+1} Q \backslash 2^{k} Q\right)}^{2},
$$

Moreover, for each $j \geq 1$,

$$
\left\|t^{j}\left(\partial_{t}\right)^{j}\left(\mathcal{S}_{t} \nabla\right) f\right\|_{L^{2}\left(\mathbb{R}^{n}\right)} \leq C_{j}\|f\|_{2}
$$

We note that by (7.11), for scalar valued $f$ we obtain from (7.14) that, for $j \geq 1$,

$$
\left\|t^{j}\left(\partial_{t}\right)^{j+1} \mathcal{S}_{t} f\right\|_{L^{2}\left(\mathbb{R}^{n}\right)} \leq C_{j}\|f\|_{2} .
$$

We further note for future reference that (7.13) can be reformulated as

$$
\left\|t^{j}\left(\partial_{t}\right)^{j}\left(\mathcal{S}_{t} \nabla\right)\left(f 1_{2^{k+1} Q \backslash 2^{k} Q}\right)\right\|_{L^{2}(Q)}^{2} \leq C_{j} 2^{-n k}\left(\frac{t}{2^{k} \ell(Q)}\right)^{2 j}\|f\|_{L^{2}\left(2^{k+1} Q \backslash 2^{k} Q\right)}^{2},
$$

so in particular,

$$
\left\|t^{2}\left(\partial_{t}\right)^{2}\left(\mathcal{S}_{t} \nabla\right)\left(f 1_{2^{k+1} Q \backslash 2^{k} Q}\right)\right\|_{L^{2}(Q)}^{2} \lesssim 2^{-(n+4) k}\|f\|_{L^{2}\left(2^{k+1} Q \backslash 2^{k} Q\right)}^{2}, \quad \text { if } t \approx \ell(Q),
$$

which by 7.11 yields also that

$$
\| t^{2}\left(\partial_{t}\right)^{3} \mathcal{S}_{t}\left(f 1_{\left.2^{k+1} Q \backslash 2^{k} Q\right)}\left\|_{L^{2}(Q)}^{2} \lesssim 2^{-(n+4) k}\right\| f \|_{L^{2}\left(2^{k+1} Q \backslash 2^{k} Q\right)}^{2}, \quad \text { if } t \approx \ell(Q) .\right.
$$

The main result of this section is the following, which is originally due to Rosen $[\mathrm{R}]$.

Theorem 7.2. Let $L$ be a t-independent complex elliptic operator as above, for which solutions of $L u=0$ and $L^{*} u=0$ satisfy the DG/N bounds (7.2) (hence also (7.3)). Then the single layer potential satisfies the following square function bound:

$$
\iint_{\mathbb{R}_{+}^{n+1}}\left|t\left(\partial_{t}\right)^{2} \mathcal{S}_{t} f(x)\right|^{2} \frac{d x d t}{t} \leq C\|f\|_{L^{2}\left(\mathbb{R}^{n}\right)}^{2} .
$$

with $C$ depending only upon $n, \lambda, \Lambda$, and the constants in (7.2) and (7.3). Analogous bounds hold in the lower half space $\mathbb{R}_{-}^{n+1}$, and for the single layer potential associated to $L^{*}$. 
This is the fundamental result concerning the layer potentials. Indeed, by AAAHK, Lemma 5.2], we have

$$
\sup _{t \neq 0}\left\|\nabla \mathcal{S}_{t} f\right\|_{L^{2}\left(\mathbb{R}^{n}\right)}^{2} \lesssim\left\|N_{*}\left(\partial_{t} \mathcal{S}_{t} f\right)\right\|_{L^{2}\left(\mathbb{R}^{n}\right)}^{2}+\int_{-\infty}^{\infty} \int_{\mathbb{R}^{n}}\left|t \partial_{t}^{2} \mathcal{S}_{t} f(x)\right|^{2} \frac{d x d t}{|t|}+\|f\|_{L^{2}\left(\mathbb{R}^{n}\right)}^{2},
$$

and also

$$
\int_{-\infty}^{\infty} \int_{\mathbb{R}^{n}}\left|t \nabla \partial_{t} \mathcal{S}_{t} f(x)\right|^{2} \frac{d x d t}{|t|} \lesssim \int_{-\infty}^{\infty} \int_{\mathbb{R}^{n}}\left|t \partial_{t}^{2} \mathcal{S}_{t} f(x)\right|^{2} \frac{d x d t}{|t|}+\|f\|_{L^{2}\left(\mathbb{R}^{n}\right)}^{2}
$$

Moreover, by [AA], we have

$$
\left\|N_{*}\left(\partial_{t} \mathcal{S}_{t} f\right)\right\|_{L^{2}\left(\mathbb{R}^{n}\right)}^{2} \lesssim \int_{-\infty}^{\infty} \int_{\mathbb{R}^{n}}\left|t \nabla \partial_{t} \mathcal{S}_{t} f(x)\right|^{2} \frac{d x d t}{|t|} .
$$

Combining these last three estimates yields, in particular, the bound

$$
\sup _{t \neq 0}\left\|\nabla \mathcal{S}_{t} f\right\|_{L^{2}\left(\mathbb{R}^{n}\right)} \lesssim\|f\|_{L^{2}\left(\mathbb{R}^{n}\right)} .
$$

Bounds for the associated double layer potential (see [AAAHK, (1.5)] for a precise definition) follow by duality, while boundedness of an appropriate non-tangential maximal function of $\nabla \mathcal{S}_{t} f$ follows by [AAAHK, Lemma 4.8]. The analogous $L^{p}$ and $H^{p}$ bounds will appear in [HMiMo].

Let us proceed now to give the proof of Theorem 7.2 .

Proof. We first make the following claim:

$$
\iint_{\mathbb{R}_{+}^{n+1}}\left|t\left(\partial_{t}\right)^{2} \mathcal{S}_{t} f(x)\right|^{2} \frac{d x d t}{t} \lesssim \iint_{\mathbb{R}_{+}^{n+1}}\left|t^{2}\left(\partial_{t}\right)^{3} \mathcal{S}_{t} f(x)\right|^{2} \frac{d x d t}{t}+\|f\|_{L^{2}\left(\mathbb{R}^{n}\right)}^{2}
$$

Indeed, the left hand side of (7.20) equals the limit, as $\delta \rightarrow 0$, of

$$
\int_{\delta}^{1 / \delta} \int_{\mathbb{R}^{n}}\left|\left(\partial_{t}\right)^{2} \mathcal{S}_{t} f(x)\right|^{2} t d x d t \lesssim \int_{\delta}^{1 / \delta} \int_{\mathbb{R}^{n}}\left|\left(\partial_{t}\right)^{2} \mathcal{S}_{t} f(x)\right|\left|\left(\partial_{t}\right)^{3} \mathcal{S}_{t} f(x)\right| t^{2} d x d t+\|f\|_{2}^{2},
$$

where we have integrated by parts in $t$, and then used (7.15), with $j=1$, to control the boundary terms. Applying "Cauchy's inequality with $\varepsilon$ 's", hiding a small term on the left hand side of the last inequality, and then letting $\delta \rightarrow 0$, we obtain (7.20). Thus, in lieu of (7.19), it is now enough to prove

$$
\iint_{\mathbb{R}_{+}^{n+1}}\left|t^{2}\left(\partial_{t}\right)^{3} \mathcal{S}_{t} f(x)\right|^{2} \frac{d x d t}{t} \leq C\|f\|_{L^{2}\left(\mathbb{R}^{n}\right)}^{2} .
$$

We therefore turn to the proof of 7.21. We shall utilize the following notation: given a vector $\vec{V}:=\left(V_{1}, \ldots, V_{n}, V_{n+1}\right) \subset \mathbb{R}^{n+1}$, we denote its "horizontal component" by

$$
V_{\|}:=\left(V_{1}, \ldots, V_{n}\right) \text {. }
$$

Similarly, the horizontal component of the $(n+1)$-dimensional gradient operator is

$$
\nabla_{\|}:=\left(\partial_{x_{1}}, \ldots, \partial_{x_{n}}\right) \text {. }
$$

For convenience, we write

$$
\left(\nabla_{y, s} u\right)(y, 0):=\left.\left(\nabla_{y, s} u(y, s)\right)\right|_{s=0} .
$$

We now define

$$
\Theta_{t}^{0}:=t^{2}\left(\partial_{t}\right)^{3} \mathcal{S}_{t}
$$


and for $f: \mathbb{R}^{n} \rightarrow \mathbb{C}^{n}$, we set

$$
\Theta_{t}^{\prime} f(x):=t^{2}\left(\partial_{t}\right)^{2} \int_{\mathbb{R}^{n}}\left(\overline{A^{*}}(y)\left(\nabla_{y, s} E\right)(x, t, y, 0)\right)_{\|} \cdot f(y) d y .
$$

It is then enough to show that the hypotheses of Theorem 6.1 are verified for this choice of $\Theta_{t}=\left(\Theta_{t}^{0}, \Theta_{t}^{\prime}\right)$, with $m=n$, where the " 0 " term corresponds to the $t=x_{n+1}$ direction. We first observe that (6.2), and 6.3) (with $\beta=2$ ), follow directly from (7.14)-(7.15) (with $j=2$ ), and (7.17)-(7.18), respectively. We shall establish the quasi-orthogonality estimate (6.4), with $m=n$, and with $H:=\left\{\nabla f: f \in \dot{L}_{1}^{2}\left(\mathbb{R}^{n}\right)\right\}$. We treat separately the contributions of $\Theta_{t}^{0} Q_{s} h^{0}$, and of $\Theta_{t}^{\prime} Q_{s} h^{\prime}$, where $h^{0} \in L^{2}\left(\mathbb{R}^{n}, \mathbb{C}\right)$, and $h^{\prime} \in H$. For the former, we use a standard "Calderón-Zygmund" argument exploiting (7.7) (with $j=3$ ), and the cancellation of the convolution kernel of $Q_{s}$; we leave the routine details to the reader. Consider now $\Theta_{t}^{\prime} Q_{s} h^{\prime}$, where $h^{\prime}=\nabla f$, with $f \in \dot{L}_{1}^{2}\left(\mathbb{R}^{n}\right)$. Recall that, modulo constants, we may identify $f \in \dot{L}_{1}^{2}\left(\mathbb{R}^{n}\right)$, with $I_{1} g$, where $g \in L^{2}\left(\mathbb{R}^{n}\right)$, and $\|g\|_{2} \approx\|\nabla f\|_{2}$, and $I_{1}=(-\Delta)^{-1 / 2}$ is the standard fractional integral operator of order 1 . Moreover, for $(x, t) \in \mathbb{R}_{+}^{n+1}$ fixed, and for $y_{n+1}:=s<t$, using that $E(x, t, y, s)=\overline{E^{*}(y, s, x, t)}$, we have (in the weak sense)

$$
\begin{aligned}
-\operatorname{div}_{y}\left(\overline{A^{*}}(y) \nabla_{y, s} E(x, t, y, s)\right)_{\|}=-\sum_{i=1}^{n} \sum_{j=1}^{n+1} \overline{\partial_{y_{i}} A_{i, j}^{*}(y) \partial_{y_{j}} E^{*}(y, s, x, t)} \\
=\sum_{j=1}^{n+1} \overline{\partial_{s} A_{n+1, j}^{*}(y) \partial_{y_{j}} E^{*}(y, s, x, t)}=-\sum_{j=1}^{n+1} \overline{A_{n+1, j}^{*}(y) \partial_{t} \partial_{y_{j}} E^{*}(y, s, x, t)},
\end{aligned}
$$

where we have used that $E^{*}(\cdot, x, t)$ is an adjoint solution away from the pole at $(x, t)$, and, in the last step, $t$-independence of the coefficients (cf. (7.5).) Thus, for $h^{\prime}=\nabla f \in H$, again using that $\overline{E^{*}(y, s, x, t)}=E(x, t, y, s)$, we have, by (7.22) and the definition of $\Theta_{t}^{\prime}$,

$$
\Theta_{t}^{\prime} Q_{s} h^{\prime}=\Theta_{t}^{\prime} \nabla_{\|} Q_{s} f=-t^{2}\left(\partial_{t}\right)^{3}\left(\mathcal{S}_{t} \nabla\right)\left(\vec{\alpha} Q_{s} I_{1} g\right),
$$

where $\vec{\alpha}:=\left(A_{1, n+1}, \ldots, A_{n+1, n+1}\right)$. By (7.14) (with $j=3$ ), since $\left\|s^{-1} Q_{s} I_{1}\right\|_{L^{2} \rightarrow L^{2}} \leq C$, we then obtain 6.4 , with $\beta=1$. We have therefore established that $\Theta_{t}^{0}$ and $\Theta_{t}^{\prime}$ satisfy all the conditions of Definition 6.1 .

It remains to construct a system $\left\{b_{Q}\right\} \subset L^{2}\left(\mathbb{R}^{n}, \mathbb{C}\right) \times H$, and a family of Borel measures $\left\{\mu_{Q}\right\}$ as in Definition 6.7 satisfying the hypotheses 6.10) 6.13) of Theorem 6.1 and also to verify hypothesis (6.14). In fact, the latter estimate is known: it has been proved in [HMaMo, Section 3], with constant $C_{1}$ depending only on dimension, ellipticity, and the DGN constants. We therefore turn to the heart of the matter, namely, the construction of the system $\left\{b_{Q}\right\}$, and the family $\left\{\mu_{Q}\right\}$, verifying (6.10)- 6.13).

Given a cube $Q \subset \mathbb{R}^{n}$, we let $x_{Q}$ denote its center, and we let

$$
X_{Q}^{ \pm}:=\left(x_{Q}, \pm \tau \ell(Q)\right)
$$

denote the upper and lower "Corkscrew points" relative to $Q$, where $\tau \in(0,1 / 8)$ is a small number at our disposal, to be chosen. Set

$$
F_{Q}(y, s):=E\left(y, s, X_{Q}^{+}\right)-E\left(y, s, X_{Q}^{-}\right),
$$

and define

$$
b_{Q}^{0}(y):=|Q|\left(\partial_{v_{A}^{-}} F_{Q}\right)(y, 0):=|Q| e_{n+1} \cdot A(y)\left(\nabla_{y, s} F_{Q}\right)(y, 0),
$$

where $e_{n+1}:=(0, \ldots, 0,1)$ is the inner unit normal to the half space $\mathbb{R}_{+}^{n+1}$ (thus, $\partial_{v_{A}^{-}}$is the outer co-normal derivative, relative to $A$, on the boundary of the lower half-space $\mathbb{R}_{-}^{n+1}$ ), 
and

$$
b_{Q}^{\prime}(y):=|Q| \nabla_{\|} F_{Q}(y, 0) .
$$

We then have, by (7.12), with $t= \pm \tau \ell(Q)$,

$$
\int_{\mathbb{R}^{n}}\left|b_{Q}\right|^{2} \leq C \tau^{-n}|Q|
$$

which is 6.10) with $C_{0} \approx \tau^{-n}$.

Next, by (7.22) and the definition of $\Theta_{t}^{\prime}$, we have that

$$
\begin{aligned}
\Theta_{t}^{\prime} b_{Q}^{\prime}(x)=t^{2}|Q|\left(\partial_{t}\right)^{3} \int_{\mathbb{R}^{n}}\left(-e_{n+1}\right) \cdot \overline{A^{*}(y)\left(\nabla_{y, s} E^{*}\right)(y, 0, x, t)} F_{Q}(y, 0) d y \\
=:-t^{2}|Q|\left(\partial_{t}\right)^{3} \int_{\mathbb{R}^{n}} \overline{\left(\partial_{v_{A^{*}}} E^{*}\right)(y, 0, x, t)} F_{Q}(y, 0) d y,
\end{aligned}
$$

so that $\partial_{v_{A^{-}}}$is the outer co-normal derivative, relative to $A^{*}$, for the lower half-space. Let $\langle\cdot, \cdot\rangle_{\mathbb{R}_{-}^{n+1}}$ denote the distributional pairing between continuous functions and measures in the lower half-space $\mathbb{R}_{-}^{n+1}$. Combining (7.24) with the definitions of $b_{Q}^{0}$ and $\Theta_{t}^{0}$, and recalling that $\overline{E^{*}(y, s, x, t)}=E(x, t, y, s)$, we have for each fixed $(x, t) \in \mathbb{R}_{+}^{n+1}$,

$$
\begin{aligned}
& \Theta_{t} b_{Q}(x)=\Theta_{t}^{0} b_{Q}^{0}(x)+\Theta_{t}^{\prime} b_{Q}^{\prime}(x) \\
& =t^{2}|Q|\left(\partial_{t}\right)^{3} \int_{\mathbb{R}^{n}}\left(\overline{E^{*}(y, 0, x, t)}\left(\partial_{v_{A}^{-}} F_{Q}\right)(y, 0)-\overline{\left(\partial_{v_{A^{*}}^{-}} E^{*}\right)(y, 0, x, t)} F_{Q}(y, 0)\right) d y \\
& =-t^{2}|Q|\left(\partial_{t}\right)^{3}\left(\left\langle\overline{E^{*}(\cdot, \cdot, x, t)}, L F_{Q}\right\rangle_{\mathbb{R}_{-}^{n+1}}-\iint_{\mathbb{R}_{-}^{n+1}} \overline{L^{*} E^{*}(y, s, x, t)} F_{Q}(y, s) d y d s\right) \\
& =t^{2}|Q|\left(\partial_{t}\right)^{3} \overline{E^{*}\left(X_{Q}^{-}, x, t\right)}=t^{2}|Q|\left(\partial_{t}\right)^{3} E\left(x, t, X_{Q}^{-}\right),
\end{aligned}
$$

where we have used the definition of $F_{Q}$, (7.4), and the fact that, in the lower half-space, $L^{*} E^{*}(\cdot, \cdot, x, t)=0=L E\left(\cdot, \cdot, X_{Q}^{+}\right)$. The preceding formal argument may be justified by introducing a smooth cut-off adapted to a ball of radius $R$, and eventually letting $R \rightarrow \infty$; there is sufficient decay at infinity to justify the limiting procedure. Therefore, by (7.6), with $j=3$, we have that for $(x, t) \in R_{Q}:=Q \times(0, \ell(Q))$,

$$
\left|\Theta_{t} b_{Q}(x)\right| \lesssim \tau^{-n-2}\left(\frac{t}{\ell(Q)}\right)^{2}
$$

Consequently,

$$
\iint_{R_{Q}}\left|\Theta_{t} b_{Q}(x)\right|^{2} \frac{d x d t}{t} \lesssim \tau^{-2 n-4}|Q| \int_{0}^{\ell(Q)}\left(\frac{t}{\ell(Q)}\right)^{4} \frac{d t}{t} \approx \tau^{-2 n-4}|Q|,
$$

which yields 6.11 with $C_{0} \approx \tau^{-2 n-4}$.

We now turn to the proofs of 6.12)-6.13). We begin by defining the measure $\mu_{Q}$. Let $\omega$ be a small, positive constant, to be chosen, and let $\phi_{Q}: \mathbb{R}^{n} \rightarrow[0,1]$ be a smooth bump function, supported in $(1+\omega) Q$, with $\phi_{Q} \equiv 1$ on $(1 / 2) Q$, where for any positive constant $\kappa$, we let $\kappa Q$ denote the cube of side length $\kappa \ell(Q)$, concentric with $Q$. Clearly, we may choose $\phi_{Q}$ so that $\left\|\nabla \phi_{Q}\right\|_{L^{\infty}\left(\mathbb{R}^{n}\right)} \leq 2 \ell(Q)^{-1}$, with $\phi_{Q} \gtrsim \omega$ on $Q$; thus (6.8) holds with $C_{0}=2$, and (6.9) holds with $C_{0} \approx 1 / \omega$. In accordance with Definition 6.7 we then set $d \mu_{Q}:=\phi_{Q} d x$. Let $\Phi_{Q}: \mathbb{R}^{n+1} \rightarrow[0,1]$ be a smooth extension of of $\phi_{Q}$, i.e., $\Phi_{Q}(x, 0)=\phi_{Q}(x)$, with $\Phi_{Q}$ supported in $I_{(1+\omega) Q}$, and $\Phi_{Q} \equiv 1$ in $I_{(1 / 2) Q}$, where in general, for any cube $Q \subset \mathbb{R}^{n}$, we let 
$I_{Q}:=Q \times(-\ell(Q), \ell(Q))$ denote the "two-sided Carleson box". We choose the extension $\Phi_{Q}$ so that

$$
\left\|\nabla \Phi_{Q}\right\|_{L^{\infty}\left(\mathbb{R}^{n+1}\right)} \leq 2 \ell(Q)^{-1}
$$

We note that, by (7.23),

$$
\int_{\mathbb{R}^{n} \backslash Q}\left|b_{Q}\right| \phi_{Q} \leq((1+\omega) Q \backslash Q)^{1 / 2}\left\|b_{Q}\right\|_{L^{2}\left(\mathbb{R}^{n}\right)}^{2} \leq C \omega^{1 / 2} \tau^{-n / 2}|Q| .
$$

Observe also that

$$
\begin{aligned}
\int_{\mathbb{R}^{n}} b_{Q}^{0} d \mu_{Q}=\int_{\mathbb{R}^{n}} b_{Q}^{0} \phi_{Q}=|Q| \int_{\mathbb{R}^{n}}\left(\partial_{v_{A}^{-}} F_{Q}\right)(y, 0) \phi_{Q}(y) d y \\
=|Q|\left(-\left\langle\Phi_{Q}, L F_{Q}\right\rangle_{\mathbb{R}_{-}^{n+1}}+\iint_{\mathbb{R}_{-}^{n+1}} A \nabla F_{Q} \cdot \nabla \Phi_{Q}\right)=:|Q|(I+I I) .
\end{aligned}
$$

By definition of $F_{Q}$ and $\Phi_{Q}$,

$$
I=\Phi_{Q}\left(X_{Q}^{-}\right)=1 .
$$

Also, by the construction of $\Phi_{Q}$, we have that

$$
\begin{aligned}
|I I| & \lesssim \frac{1}{\ell(Q)} \iint_{I_{(1+\omega) Q} \backslash I_{(1 / 2) Q}}\left|\nabla F_{Q}\right| \\
& \lesssim \ell(Q)^{(n-1) / 2}\left(\iint_{I_{(1+\omega) Q} \backslash I_{(1 / 2) Q}}\left|\nabla F_{Q}\right|^{2}\right)^{1 / 2} \lesssim \ell(Q)^{(n-3) / 2}\left(\iint_{I_{2 Q} \backslash I_{(1 / 4) Q}}\left|F_{Q}\right|^{2}\right)^{1 / 2} \\
& =\ell(Q)^{(n-3) / 2}\left(\iint_{I_{2 Q} \backslash I_{(1 / 4)}}\left|\int_{-\tau \ell(Q)}^{\tau \ell(Q)} \partial_{t} E\left(y, s, x_{Q}, t\right) d t\right|^{2} d y d s\right)^{1 / 2} \lesssim \tau,
\end{aligned}
$$

where the implicit constants depend only on dimension, ellipticity, and the DGN constants, and where in the last three steps we have used Caccioppoli's inequality, the definition of $F_{Q}$, and (7.6) with $j=1$. Combining our estimates for terms $I$ and $I I$, we obtain

$$
\operatorname{Re} \int_{\mathbb{R}^{n}} b_{Q}^{0} d \mu_{Q} \geq|Q|(1-C \tau) .
$$

In conjunction with (7.25), the latter bound implies

$$
\operatorname{Re} \int_{Q} b_{Q}^{0} d \mu_{Q} \geq|Q|\left(1-C \tau-C \omega^{1 / 2} \tau^{-n / 2}\right) \geq \frac{1}{2}|Q|,
$$

if we set $\omega:=\tau^{n+2}$, and choose $\tau$ sufficiently small. Thus, we obtain (6.12) with $\sigma=1 / 2$.

Finally, we verify (6.13). By definition of $b_{Q}^{\prime}, \mu_{Q}, \phi_{Q}$, and $F_{Q}$, we have

$$
\begin{aligned}
\left|\int_{\mathbb{R}^{n}} b_{Q}^{\prime} d \mu_{Q}\right|=|Q| & \left|\int_{\mathbb{R}^{n}} \nabla_{y} F_{Q}(y, 0) \phi_{Q}(y) d y\right|=|Q|\left|\int_{\mathbb{R}^{n}} F_{Q}(y, 0) \nabla_{y} \phi_{Q}(y) d y\right| \\
& \leq C \ell(Q)^{n-1} \int_{(1+\omega) Q \backslash(1 / 2) Q}\left|\int_{-\tau \ell(Q)}^{\tau \ell(Q)} \partial_{t} E\left(y, 0, x_{Q}, t\right) d t\right| d y \leq C \tau|Q|,
\end{aligned}
$$

where in the last step we have used (7.6) with $j=1$. Combining the latter estimate with (7.25), we have

$$
\left|\int_{Q} b_{Q}^{\prime} d \mu_{Q}\right| \leq C\left(\tau+\omega^{1 / 2} \tau^{-n / 2}\right)|Q| \leq C \tau|Q|,
$$


by our choice of $\omega=\tau^{n+2}$. Since the constant $\tau$ is at our disposal (cf. Remark 6.4), we obtain 6.13), with $\eta \approx \tau$. This concludes the proof of our application, Theorem 7.2

\section{Appendix: proof of the generalized Christ-Journé $T 1$ Theorem for sQuare functions}

In this Appendix, we give the proofs of Theorems 3.6 and 4.3. In fact, it will suffice to prove the latter, since taking $H=L^{2}$ then yields the former. The proof will follow that of the $T 1$ theorem of $[\overline{C J}]$; indeed, the lack of pointwise kernel bounds, and the fact that our square function acts only on elements of $H$, do not present serious obstacles.

Proof of Theorem 4.3 . We consider

$$
\iint_{\mathbb{R}_{+}^{n+1}}\left|\Theta_{t} h(x)\right|^{2} \frac{d x d t}{t},
$$

with $h \in H$, where $H$ is a subspace of $L^{2}\left(\mathbb{R}^{n}, \mathbb{C}^{m}\right)$. Let us set

$$
d \mu(x, t):=\left|\Theta_{t} 1(x)\right|^{2} \frac{d x d t}{t}
$$

so that, by hypothesis, we have the Carleson measure estimate

$$
\|\mu\|_{C}:=\sup _{Q} \frac{1}{|Q|} \iint_{R_{Q}} d \mu(x, t)<\infty .
$$

We follow the familiar idea of Coifman-Meyer to write

$$
\Theta_{t} h=\left(\Theta_{t}-\left(\Theta_{t} 1\right) A_{t} P_{t}\right) h+\left(\Theta_{t} 1\right) A_{t} P_{t} h=: R_{t} h+\left(\Theta_{t} 1\right) P_{t} h,
$$

where as usual, $A_{t}$ is the dyadic averaging operator, and $P_{t}$ is a nice approximate identity. By Carleson's embedding lemma, and the well known non-tangential estimate for $A_{t} P_{t} h$,

$$
\iint_{\mathbb{R}_{+}^{n+1}}\left|\left(\Theta_{t} 1\right) A_{t} P_{t} h(x)\right|^{2} \frac{d x d t}{t} \lesssim\|\mu\|_{C}\|h\|_{2}^{2},
$$

as desired (we remark that for this term, the estimate holds for all $h \in L^{2}\left(\mathbb{R}^{n}, \mathbb{C}^{m}\right)$.) Moreover,

$$
\iint_{\mathbb{R}_{+}^{n+1}}\left|R_{t} h(x)\right|^{2} \frac{d x d t}{t} \lesssim\|h\|_{2}^{2}, \quad \forall h \in H,
$$

by Corollary 3.12 ; indeed, the present $R_{t}$ is precisely the same (up to a minus sign) as $R_{t}^{(2)}$ considered in (3.24).

\section{REFERENCES}

[AAAHK] M. A. Alfonseca, P. Auscher, A. Axelsson, S. Hofmann, and S. Kim, Analyticity of Layer Potentials and $L^{2}$ Solvability of Boundary Value Problems for Divergence Form Elliptic Equations with Complex $L^{\infty}$ Coefficients, Advances in Math. 226 (2011), 4533-4606..

[A] P. Auscher, On necessary and sufficient conditions for $L^{p}$-estimates of Riesz Transforms associated to elliptic operators on $\mathbb{R}^{n}$ and related estimates, Memoirs of the American Mathematical Society, volume 186, number 871, March 2007.

[A2] P. Auscher, Regularity theorems and heat kernel for elliptic operators. J. London Math. Soc. (2) 54 (1996), no. 2, 284-296.

[AA] P. Auscher and A. Axelsson, Weighted maximal regularity estimates and solvability of non-smooth elliptic systems I, Invent. Math. 184 (2011), 47-115.

[AAMc] P. Auscher, A. Axelsson, and A. McIntosh, Solvability of elliptic systems with square integrable boundary data, Ark. Mat. 48 (2010), 253-287.

[AHLMcT] P. Auscher, S. Hofmann, M. Lacey, A. McIntosh, and P. Tchamitchian, The solution of the Kato Square Root Problem for Second Order Elliptic operators on $\mathbb{R}^{n}$, Annals of Math. 156 (2002), 633654. 
[AHMTT] P. Auscher, S. Hofmann, C. Muscalu, T. Tao, C. Thiele, Carleson measures, trees, extrapolation, and $T(b)$ theorems, Publ. Mat. 46 (2002), no. 2, 257325.

[AMcT] P. Auscher, A. McIntosh and P. Tchamitchian, Heat kernel of complex elliptic operators and applications, J. Funct. Anal. 152(1998), 22-73.

[AR] P. Auscher, E. Routin, Local Tb Theorems and Hardy inequalities, http://arxiv.org/abs/1011.1747

[AT] P. Auscher, P. Tchamitchian, Square root problem for divergence operators and related topics, Astérisque Vol. 249(1998)m société Mathématique de France.

[AY] P. Auscher, Qi Xiang Yang, BCR algorithm and th T(b) theorem, Publ. Math.53 (2009), no. 1, 179196.

$[\mathrm{Ch}] \mathrm{M}$. Christ, $A T(b)$ theorem with remarks on analytic capacity and the Cauchy integral, Colloquium Mathematicum LX/LXI (1990) 601-628.

[CJ] M. Christ and J.-L. Journé, Polynomial growth estimates for multilinear singular integral operators. Acta Math. 159 (1987), no. 1-2, 51-80.

[CMS] R. R. Coifman, Y. Meyer, and E. M. Stein, Some New Function Spaces and Their Applications to Harmonic Analysis, J. Funct. Anal. 62 (1985), 304-335.

[CR] R.Coifman and R. Rochberg, Another characterization of B.M.O., Proc. Amer. Math. Soc 79,249-254.

[C-UMP] D. Cruz-Uribe, J.M Martell and C. Pérez, Extensions of Rubio de Francia's extrapolation theorem,Proceedings of the 7th International Conference on Harmonic Analysis and Partial Differential Equations (El Escorial 2004), Collect. Math.2006,195-231..

[DJS] G. David, J.-L. Journé, and S. Semmes, Opérateurs de Calderón-Zygmund, fonctions para-accrétives et interpolation, Rev. Mat. Iberoamericana 1 156, 1985.

[D] E.B. Davies, Uniformly elliptic operators with measurable coefficients, J. Funct. Anal. 132 (1995), 141-169.

[DRdeF] J. Duoandikoetxea, J. L. Rubio de Francia, Maximal and singular integral operators via Fourier transform estimates, Inventiones Math 84 (1986), 541-561.

[DeG] E. De Giorgi, Sulla differenziabilità e l'analiticità delle estremali degli integrali multipli regolari, Mem. Accad. Sci. Torino. Cl. Sci. Fis. Mat. Nat. (3) 3 (1957) 25-43.

[FKP] R. Fefferman, C. Kenig and J. Pipher, The theory of weights and the Dirichlet problem for elliptic equations, Ann. of Math. (2) 134 (1991), no. 1, 65-124.

[FS] C. Fefferman and E. M. Stein, $H^{p}$ spaces of several variables. Acta Math. 129 (1972), no. 3-4, 137-193.

[GR] J. Garcia Cuerva and J. Rubio de Francia, Weighted norm inequalities and related topics, North-Holland, 1985.

[GM] A. Grau de la Herrán and M. Mourgoglou, A Tb theorem for square functions in domains with AhlforsDavid regular boundaries, to appear in the Journal of Geometric Analysis.

[H1] S. Hofmann, Local T(b) theorems and application in PDE. Harmonic analysis and partial differential equations 29-52, Contemp. Math, 505, Amer. Math. Soc., Providence, RI, 2010.

[H2] S. Hofmann, A Local Tb Theorem For Square Functions, Perspectives in partial differential equations, harmonic analysis and applications, 175185, Proc. Sympos. Pure Math., 79, Amer. Math. Soc., Providence, RI, 2008.

[H3] S. Hofmann, A proof of the local Tb theorem for standard Calderon-Zygmund operators, unpublished manuscript (2007), http://arxiv.org/abs/0705.0840.2

[H4] S. Hofmann, Local Tb Theorem For Square Functions and Application in PDE, Proceedings of the ICM Madrid 2006.

[HKMP] S. Hofmann, C. Kenig, S. Mayboroda, and J. Pipher, Square function/Non-tangential maximal estimates and the Dirichlet problem for non-symmetric elliptic operators, preprint.

[HK] S. Hofmann and S. Kim, The Green function estimates for strongly elliptic systems of second order, Manuscripta Math. 124 (2007), no. 2, 139-172.

[HK2] S. Hofmann and S. Kim, Gaussian estimates for fundamental solutions to certain parabolic systems, Publ. Mat. 48 (2004), no. 2, 481-496.

[HLMc] S. Hofmann, M. Lacey and A. McIntosh, The solution of the Kato problem for divergence form elliptic operators with Gaussian heat kernel bounds, Annals of Math. 156 (2002), pp 623-631.

[HMaMo] S. Hofmann, S. Mayboroda, and M. Mourgoglou, $L^{p}$ and endpoint solvability results for divergence form elliptic equations with complex $L^{\infty}$ coefficients, preprint.

[HMar] S.Hofmann and J.M. Martell, Uniform rectifiability and harmonic measure I: Uniform rectifiability implies Poisson kernels in $L^{p}$, preprint.

[HMarUT] S.Hofmann, J.M. Martell and I. Uriarte-Tuero, Uniform rectifiability and harmonic measure II: Poisson kernels in $L^{p}$ imply uniform rectfiability, preprint.

[HMc] S. Hofmann and A. McIntosh, The solution of the Kato problem in two dimensions, Proceedings of the Conference on Harmonic Analysis and PDE held in El Escorial, Spain in July 2000, Publ. Mat. Vol. extra, 2002 pp. 143-160. 
[HMiMo] S. Hofmann, M. Mitrea, and A. Morris, manuscript in preparation.

[HM] S. Hofmann and M. Mitrea, Boundary Value Problems and the Method of Layer Potentials for Elliptic Operators with $L^{\infty}$ Coefficients preprint.

[HyM] T. Hytönen, H. Martikainen, On general local Tb Theorems, http://arxiv.org/abs/1011.0642

[HyN] T. Hytönen, F. Nazarov, The local Tb theorem with rough test functions, http://arxiv.org/abs/1206.0907.

[McM] A. McIntosh and Y. Meyer, Algèbres dopérateurs définis par des intégrales singulières, C. R. Acad. Sci. Paris 301 Série 1 395397, 1985.

[Me] N. G. Meyers, Mean oscillation over cubes and Hölder continuity, Proc. Amer. Math. Soc. 15 (1964) 717-721.

[Me2] N. G. Meyers, An $L^{p}$ estimate for the gradient of solutions of second order elliptic divergence equations, Ann. Scuola Norm. Sup. Pisa 17 (1963), 189-206.

[M] J. Moser, On Harnack's theorem for elliptic differential equations, Comm. Pure Appl. Math. 14 (1961) $577-$ 591.

[N] J. Nash, Continuity of solutions of parabolic and elliptic equations, Amer. J. Math. 80 (1958) 931-954.

[NTV] F. Nazarov, S. Treil and A. Volberg, Accretive system Tb-theorems on nonhomogeneous spaces, Duke Math. J. 113 (2002), no. 2, 259312.

[R] A. Rosen, Layer potentials beyond singular integral operators, preprint

[Se] S. Semmes, Square function estimates and the T(b) Theorem. Proc. Amer. Math. Soc. 110 (1990), no. 3, 721726.

[St] E. M. Stein, Harmonic Analysis: Real-Variable Methods, Orthogonality, and Oscilatory Integrals, Princeton, NJ: Princeton University Press, 1993.

[St2] E.M. Stein, Singular integrals and differentiability properties of functions, Princeton, NJ: Princeton University Press, 1971.

[SW] E.M. Stein and G. Weiss, Interpolation of operators with change of measures, Trans. Amer. Math. Soc. 87,(1958), 159-172.

[TY] C.Tan, L. Yan, Local Tb theorem on spaces of homogeneous type, Z. Anal. Anwend.28 (2009), no.3, 333347.

[V] G. Verchota, Layer potentials and regularity for the Dirichlet problem for Laplace's equation in Lipschitz domains. J. Funct. Anal. 59 (1984), no. 3, 572-611.

DEPARTMENT OF MATHEMATICS AND STATISTICS, UNIVERSITY OF HELSINKI, FINLAND

E-mail address: ana.grau@helsinki.fi

DEPARTMENT OF MATHEMATICS, UNIVERSITY OF MISSOURI, COLUMBIA

E-mail address: hofmanns@missouri.edu 